PREPARED FOR THE U.S. DEPARTMENT OF ENERGY, UNDER CONTRACT DE-AC02-76CH03073

PPPL-3573

PPPL-3573

UC-70

Plasma Profile and Shape Optimization for the Advanced Tokamak Power Plant, ARIES-AT

C.E. Kessel, T.K. Mau, S.C. Jardin, and F. Najmabadi

June 2001

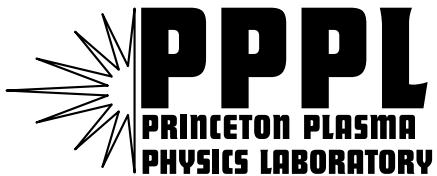

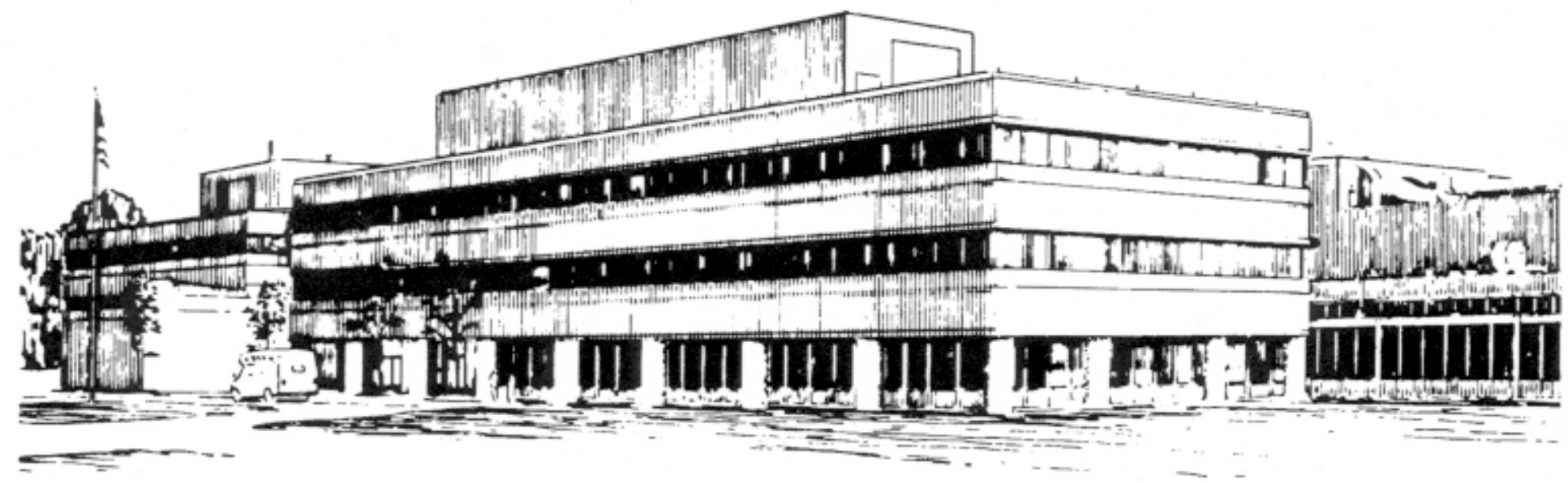

PRINCETON PLASMA PHYSICS LABORATORY PRINCETON UNIVERSITY, PRINCETON, NEW JERSEY 


\section{PPPL Reports Disclaimer}

This report was prepared as an account of work sponsored by an agency of the United States Government. Neither the United States Government nor any agency thereof, nor any of their employees, makes any warranty, express or implied, or assumes any legal liability or responsibility for the accuracy, completeness, or usefulness of any information, apparatus, product, or process disclosed, or represents that its use would not infringe privately owned rights. Reference herein to any specific commercial product, process, or service by trade name, trademark, manufacturer, or otherwise, does not necessarily constitute or imply its endorsement, recommendation, or favoring by the United States Government or any agency thereof. The views and opinions of authors expressed herein do not necessarily state or reflect those of the United States Government or any agency thereof.

\section{Availability}

This report is posted on the U.S. Department of Energy's Princeton Plasma Physics Laboratory Publications and Reports web site in Calendar Year 2001. The home page for PPPL Reports and Publications is: http://www.pppl.gov/pub_report/

DOE and DOE Contractors can obtain copies of this report from:

U.S. Department of Energy

Office of Scientific and Technical Information

DOE Technical Information Services (DTIS)

P.O. Box 62

Oak Ridge, TN 37831

Telephone: (865) 576-8401

Fax: (865) 576-5728

Email: reports@adonis.osti.gov

This report is available to the general public from:

National Technical Information Service

U.S. Department of Commerce

5285 Port Royal Road

Springfield, VA 22161

Telephone: 1-800-553-6847 or

(703) 605-6000

Fax: (703) 321-8547

Internet: http://www.ntis.gov/ordering.htm 


\title{
Plasma Profile and Shape Optimization for the Advanced Tokamak Power Plant, ARIES-AT
}

\author{
C. E. Kessel ${ }^{1}$, T. K. Mau' ${ }^{2}$, S. C. Jardin' ${ }^{1}$, F. \\ Najmabadi ${ }^{2}$
}

\begin{abstract}
An advanced tokamak plasma configuration is developed based on equilibrium, ideal MHD stability, bootstrap current analysis, vertical stability and control, and poloidal field coil analysis. The plasma boundaries used in the analysis are forced to coincide with the $99 \%$ flux surface from the freeboundary equilibrium. Using an accurate bootstrap current model and external current drive profiles from ray tracing calculations in combination with optimized pressure profiles, $\beta_{N}$ values above 7.0 have been obtained. The minimum current drive requirement is found to lie at a lower $\beta_{N}$ of 5.4. The external kink mode is stabilized by a tungsten shell located at 0.33 times the minor radius and a feedback system. Plasma shape optimization has led to an elongation of 2.2 and triangularity of 0.9 at the separatrix. Vertical stability could be achieved by a combination of tungsten shells located at 0.33 times the minor radius and feedback control coils located behind the shield. The poloidal field coils were optimized in location and current, providing a maximum coil current of 8.6 MA. These developments have led to a simultaneous reduction in the power plant major radius and toroidal field.
\end{abstract}

\section{Introduction}

The simultaneous achievement of high $\beta$ (at high plasma current), high bootstrap fraction, and the transport suppression consistent with these features was first shown in ref[1]. An overview of experimental and theoretical results, excluding the most recent, was given in ref[2]. The reversed shear configuration for the tokamak has the potential to be an economical power plant [2], and its features, referred to as the advanced tokamak, are being pursued in several tokamak experiments[5]-[11]. Previous work reported in ref[2] obtained attractive $\beta$ values and reasonable current

\footnotetext{
${ }^{1}$ Princeton Plasma Physics Laboratory, P.O. Box 451, Princeton, NJ 08543 USA

${ }^{2}$ Center for Energy Research, University of California San Diego, 9500 Gilman Drive, La Jolla, CA 93037 USA
} 
drive requirements. However, it is of interest to further improve such configurations to understand the potential benefits and identify the highest leverage research areas. The present studies will show that accurate bootstrap models are necessary to determine MHD stability and current drive requirements. In addition, requiring the plasma boundary to coincide with the $99 \%$ flux surface of the free-boundary plasma enforces consistency and has led to higher $\beta$ limits. The plasma pressure profile is optimized to provide high ballooning $\beta$ limits and bootstrap current alignment simultaneously. Plasma shaping has been utilized, within engineering constraints, to increase the $\beta$, both through increases in $\beta_{N}\left(\beta_{N}=\beta \mathrm{aB} T / \mathrm{I}_{p}\right.$, a $=$ minor radius, $\mathrm{B}_{T}=$ toroidal field, and $\mathrm{I}_{p}=$ plasma current) and plasma current. Vertical stability and control analysis have found a reasonable solution for the passive stabilizer and feedback control power. Poloidal field (PF) coil optimization has been performed and meets all engineering constraints.

\section{Equilibrium and Stability Studies}

The fixed boundary flux-coordinate equilibrium code JSOLVER [12] is used with 257 flux surfaces and 257 theta points from 0 to $\pi$. The $n=\infty$ ballooning stability is analized with BALMSC [13], and PEST2 [14] is used for low-n external kink stability. For all cases reported here, a conducting wall is assumed to stabilize the external kink modes, and this wall location is determined. In addition, $n=0$ vertical stability is assessed with Corsica [15].

The final optimized reversed shear plasma is shown in Fig. 1, with various equilibrium profiles, and global plasma parameters are given in Table 1, under ARIESAT, where they are compared to ARIES-RS[2]. The plasma boundaries used in the fixed-boundary equilibrium calculations are taken from free-boundary equilibria at the $99 \%$ poloidal magnetic flux surface, in which $q_{95}$ is kept $\geq 3.0$. We attempt to use a flux surface as close to the diverted plasma separatrix as possible, limited by the ability of the fixed-boundary equilibrium and ideal MHD stability codes to handle the angular boundary near the X-point. This approach can have a strong impact on the calculated stable $\beta$ values due to progressively stronger plasma shaping as one approaches the separatrix. Plotted in Fig. 2 are the elongation and triangularity for a series of flux surfaces from the $95 \%$ to $99.5 \%$. The cases shown have a plasma internal self-inductance (1i) of 0.46 . Both elongations and triangularities would increase with lower li, and decrease with higher li. Later the impact of stronger shaping will be discussed. In addition, this provides strict consistency between the PF coil generated free-boundary equilibrium and the fixed-boundary equilibria, which is important due to the non-analytic boundaries that are generated with actual coils.

In order to calculate free-boundary equilibria for the advanced plasmas being considered, it was necessary to make modifications to the methods used. The free- 
boundary equilibrium equation to be solved is given by the Grad-Shafranov equation,

$$
\begin{gathered}
\Delta^{*} \psi=-\mu_{\circ} R j_{\phi} \\
j_{\phi}=-R \frac{d p}{d \psi}-\frac{1}{2 \mu_{o} R} \frac{d g^{2}}{d \psi}
\end{gathered}
$$

where $\psi$ is the poloidal flux function (equal to the actual poloidal flux divided by $2 \pi), j_{\phi}$ is the toroidal current density, $p$ is the pressure, and $g$ is the toroidal field function (equal to $R B_{\phi}$ ). For reversed shear plasmas at high pressure the two terms in the definition of $j_{\phi}$ have opposite signs cancelling to some degree. Physically, the toroidal current density is low in the plasma core due to the hollow current profile, but is also shifting to the outboard side as the pressure increases. This cancellation between the two terms is critical to obtaining proper force balance, and is not easily achieved for arbitrary choices of the functions $p(\psi)$ and $g(\psi)$. In order to produce these equilibria and have them represent the desirable fixed boundary configurations with high $\beta$ and high bootstrap current fractions, we recast the toroidal current density in terms of the pressure and parallel current density,

$$
j_{\phi}=-R \frac{d p}{d \psi}\left(1-\frac{B_{\phi}^{2}}{\left\langle B^{2}\right\rangle}\right)+B_{\phi} \frac{\langle j \cdot B\rangle}{\left\langle B^{2}\right\rangle}
$$

The first term is the combination of the Phirsch-Schluter and diamagnetic currents, and the second term is any driven parallel current that exists (i.e. ohmic, bootstrap, RFCD). Now both terms are positive and don't require cancellation at high pressure, and specifiying the parallel current density is how fixed boundary equilibria are calculated in JSOLVER. This specification of the $j_{\phi}$ requires flux surface averages to be calculated at each iteration, which slows the calculation down, however, with this approach the pressure, current, and safety factor profiles can be accurately represented between fixed and free-boundary equilibria. In addition, one can obtain difficult equilibria not accessible with the original formulation.

There are two types of fixed boundary equilibrium calculations used, target equilibria and self-consistent equilibria. Target equilibria have the total parallel current density and pressure profile prescribed. These are used to scan MHD stability more efficiently for plasma shape and profile optimization. The bootstrap current profile is calculated [16] at the end of the equilibrium calculation, and is monitored for alignment with the prescribed current profile. Self-consistent equilibria have only the pressure profile and the parallel current density profiles from current drive sources (i.e. FWCD and LHCD) prescribed. The bootstrap current density is calculated at each iteration of the equilibrium, which is added to the current drive sources to provide the total parallel current density. These equilibria are used to develop final configurations, by prescribing the required current drive profiles and interating with the actual current drive deposition profiles from ray tracing calculations reported in $\operatorname{ref}[17]$. 


\section{Current Profiles and Bootstrap Current}

The parallel current density profile used in target equilibrium calculations is given by the following form,

$$
\frac{\langle j \cdot B\rangle}{\langle B \cdot \nabla \phi\rangle}=j_{0}(1-\hat{\psi})+j_{1} \frac{d^{2} \hat{\psi}^{a_{j}}(1-\hat{\psi})^{b_{j}}}{\left(\hat{\psi}-\hat{\psi}_{0}\right)+d^{2}}
$$

where $\hat{\psi}$ is the normalized poloidal flux that is zero at the magnetic axis and 1.0 at the plasma edge. The parameters are chosen to generate a hollow current profile. The particular form is chosen by considering the ability to reproduce the profile shape with bootstrap current and the need for external current drive. The location of the $q_{\text {min }}$ (minimum safety factor) is chosen as close to the plasma edge as possible to obtain high ballooning $\beta$-limits, while avoiding the degradation of the magnetic shear near the plasma edge, and to stay within external current drive limitations (i.e. LH current penetration).

For the self-consistent equilibrium calculations only the external current drive profiles are input, and they are described by terms that are the same as the second term in Eqn. 4. An example of these appears in Fig. 1, where the parallel current density is shown, and the on-axis FWCD and off-axis LHCD profiles are shown. The bootstrap current is calculated self-consistently in Fig. 1.

It was found by comparing full collisional bootstrap calculations[16] to the single ion collisionless calculation[18], that the external current drive required in the two cases is quite different. A comparison was made where two equilibria with $\approx 100 \%$ bootstrap current, all other plasma parameters the same, and $\beta_{N}$ of 5.35 , shown in Fig. 3. The collisionless case ignores the effect of decreasing temperature near the plasma edge, which would strongly reduce the bootstrap current there, and has an overall shift of the current profile outward. This causes $q_{\min }$ to be further out in minor radius, which for a fixed pressure profile, will give a higher ballooning $\beta$-limit. The collisionless model is misleading because it indicates that no off-axis current drive is required to achieve ballooning stability at this $\beta_{N}$. The collisional model, on the other hand, would require off-axis current drive of about $10 \%$ of the total plasma current to obtain ballooning stability at this $\beta_{N}$. The difference in the location of $q_{\text {min }}$ for the two bootstrap models is critical to assessing the ideal $\beta$-limit and the magnitude of off-axis current drive. The use of a collisionless model should be avoided in configuration development because of its overly optimistic predictions for bootstrap current near the plasma edge.

The bootstrap current calculation requires knowledge of the pressure profile and either the density or temperature profile. In the present work particle and energy transport calculations were not done. Rather, the density and temperature profiles were constrained to have their dominant gradients in the vicinity of the $q_{\text {min }}$ consistent with the presence of an internal transport barrier (ITB)[5]-[11]. This approach was chosen due to the widely varying characteristics observed on various reversed shear tokamak experiments, with the exception of this dominant gradient. Beyond 
this, the profiles are chosen to optimize the bootstrap current alignment, and typical profiles for these plasmas are shown in Fig. 1. Ref[17] presents a comparison of the assumed profiles and those from GLF23 theoretical predictions indicating reasonable agreement. The density and temperature profiles assumed in this study show gradients that are spread out more than is observed experimentally, and may require some form of ITB control to achieve, but this is beyond the scope of the present work.

\section{Pressure Profile Optimization}

The pressure profile is described by the following,

$$
p(\psi)=p_{\circ}\left[c_{1}\left(1-\hat{\psi}^{b_{1}}\right)^{a_{1}}+c_{2}\left(1-\hat{\psi}^{b_{2}}\right)^{a_{2}}\right]
$$

where the coefficients are chosen to reduce the pressure gradient in the region outside the $q_{\min }$ to improve ballooning stability, and to provide bootstrap current alignment to reduce the magnitude of external current drive. The pressure gradient is zero at the plasma edge for these profiles, making these typical of an L-mode edge. Previously[2], the pressure was parameterized by a simpler form,

$$
p(\psi)=p_{o}\left(1-\hat{\psi}^{b_{1}}\right)^{2}
$$

which was restrictive in optimizing both the ballooning stability and bootstrap current simultaneously.

A sequence of pressure profiles was determined, using Eqn. 5, that had progressively higher $\beta$-limits, due to a progressively smaller pressure gradient in the ballooning unstable region. This is shown in Fig. 4, where the pressure gradient is plotted as a function of poloidal flux. The region of ballooning instability is noted, and the remaining part of the pressure profile is made to provide bootstrap alignment. The smaller pressure gradients near the plasma edge, to increase $\beta$, led to smaller bootstrap current there causing higher external current to be required to provide stability. In addition, the highest $\beta$ 's led to excessive bootstrap current in the plasma core, which requires a broader density profile to eliminate it, exacerbating the current drive problem near the plasma edge. These two factors cause the current drive requirement to begin increasing as $\beta$ rises sufficiently high. At lower $\beta$ values there is insufficient bootstrap current to provide a large bootstrap fraction, within our transport constraints, leading to large current drive requirements.

The full stabilization of the external kink mode requires either plasma rotation[20] or feedback control[21]. Plasma rotation is difficult to provide for reactor size plasmas so the feedback approach is taken. For this approach the shell is necessary to slow the mode growth rate to time scales which the feedback coils can respond with reasonable power. This is discussed in ref[17]. If no transport constraints are imposed, one can find very broad temperature and very peaked density profile solutions 
that can provide the bootstrap current at low $\beta$, but these are considered unphysical. Due to these effects the required externally driven current as a function of $\beta_{N}$ shows a minimum, and this is shown in Fig. 5. From ray tracing calculations[17] the $\beta_{N}$ of 6.0 was chosen as the best tradeoff between maximizing $\beta$ and minimizing current drive power.

\section{Plasma Shape Optimization}

The plasma triangularity and elongation have a strong impact on the ideal MHD stability. In addition to providing higher $\beta_{N}$ values they also allow higher plasma current to be driven in the plasma for $q_{95}$ constrained to be $\geq 3.0$.

The plasma triangularity has been limited by the need to provide a sufficiently long slot divertor on the inboard side to obtain a detached plasma and radiate the power there. In a power plant, the divertor slot must cut through blanket and shielding, which allows neutrons to penetrate close to the superconducting toroidal field magnet. This sets a limit on the triangularity (or the angle that the separatrix flux line can make with the inboard wall) to provide neutron sheilding. However, recently both experimental and theoretical results indicate that the inboard plasma detachment can be obtained without a slot, although a short slot is still used to disperse the heat load. This allows us to take advantage of higher triangularities. To assess the benefits, a scan of the triangularity was done, for two elongations. For this scan the plasma boundary is given analytically, the edge safety factor is held fixed at 3.5 , the aspect ratio is 4.0 , and one of the pressure profiles described above was used. Shown in Fig. 6 and 7 is the $\beta_{N}$ and $\beta$ as a function of triangularity, for $n=\infty$ ballooning modes. Included is the result given earlier[2], with a more restrictive pressure profile, Eqn. 6. It is clear that $\beta_{N}$ improves with higher triangularity, but a rollover occurs beyond a triangularity of 0.65 . However, the $\beta$ continues to rise due to the continued increase in the plasma current. The combination of higher elongation creates an even stronger increase in $\beta$.

The plasma elongation is limited by the $n=0$ vertical instability, through the ability to provide conducting structure close enough to the plasma and a vertical position feedback system with reasonable power. For power plant designs the location of a conducting material is limited to lie outside the blanket, and typically resides in the region between the blanket and shield. The plasma elongation can substantially increase $\beta$ due to a $\left(1+\kappa^{2}\right)$ scaling. Recently, optimization of the blanket[19] has allowed the conducting structure to be moved closer to the plasma, and actually be in the blanket, although, it must exist in a very high temperature environment $\left(\geq 1000^{\circ} \mathrm{C}\right)$. The benefits of increasing the plasma elongation above the values previously found[2] for a conducting structure behind the blanket were examined. As for the triangularity scan, analytic plasma boundaries were used, with a range of triangularities between 0.4 and 0.85 , aspect ratio of 4.0 , the edge safety factor fixed at 3.5, and one of the pressure profiles described by Eqn. 5. Shown in 
Fig. 8 and 9 are $\beta_{N}$ and $\beta$ as a function of elongation, for $n=\infty$ ballooning modes. The $\beta_{N}$ shows a decrease with increasing elongation at lower triangularity, and the opposite at higher triangularity. Although, at higher triangularity the increase in $\beta_{N}$ turns over and begins to decrease. The point where the $\beta_{N}$ increase turns over moves to higher elongations as the triangularity is increased. As was seen for the triangularity scan, the $\beta$ values continue to increase regardless of the structure in the $\beta_{N}$ versus elongation curves, because the increase in plasma current is so strong. The slope of the increase in $\beta$ versus elongation continues to improve with higher triangularity over the whole range.

The low-n external kink stability was also analized, and is shown in Fig. 10. Here the marginal wall location is given as a function of elongation for toroidal mode numbers from 1 to 6 . The wall locations are only resolved to 0.025 times the minor radius. Higher toroidal mode numbers require closer walls for stabilization, and all mode number wall locations move much closer to the plasma as the elongation exceeds a value of 2.3. The triangularity for this case was fixed at 0.7 . In order to observe the impact of triangularity on the kink mode wall stabilization, three other values were analized; 0.4, 0.55, and 0.85. Shown in Fig. 11 are the marginal wall locations for toroidal mode number $n=1$, as a function of elongation. It is clear that higher triangularity results in marginal wall locations that are farther from the plasma at the lower elongations, however, the stability at the highest elongations is rapidly degrading. Shown in Fig. 12 are the marginal wall locations for toroidal mode numbers $n=1-5$, at a fixed elongation, and varying triangularity, showing that the improvement with triangularity persists at higher $n$. As was pointed out in ref[1], when a stablizing wall is present there is typically a toroidal mode number greater than 1 that is the most limiting to $\beta_{N}$. Although the curves in Fig. 10 do not indicate a minimum, the analysis for a triangularity of 0.7 , and elongation of 2.2 was extended to toroidal mode numbers up to 9 to resolve this limiting mode. Shown in Fig. 13 is the marginal wall location as a function of toroidal mode number, at two different $\beta_{N}$ values, with $\beta_{N}$ corresponding to the final plasma configuration. As the pressure is increased the $\beta$-limiting toroidal mode number moves to higher values and the wall must move closer to the plasma to stablize all $\mathrm{n}$.

Based on the plasma shape analysis, values for the elongation and triangularity at the separatrix were chosen to be 2.2 and 0.9 , respectively. This integrated the ballooning and kink stability behavior, requiring a shell for the kink mode stabilization at 0.33 times the minor radius, and avoiding the rapid degradation in kink stability at higher elongations. As will be shown in the next section, the shell for vertical stability is placed at the same location, and therefore provides full coverage on the plasma outboard side as required for kink stabilization. The plasma elongation choice was also dependent on vertical stability analysis that follows. 


\section{Axisymmetric Stability}

The plasma elongation is ultimately limited by the vertical instability, through the ability to locate conducting structure sufficiently close to the plasma and provide a feedback control system with reasonable power. Due to the high leverage of plasma elongation in increasing $\beta$, scans were done to determine the plasma growth rate, and stability factor as a conducting shell is moved progressively further away from the plasma, for various plasma elongations. The stability factor is defined as,

$$
f_{s}=1+\tau_{g} / \tau_{L / R}
$$

where $\tau_{g}$ is the vertical instability growth time, and $\tau_{L / R}$ is the longest up-down asymmetric time constant of the surrounding structure. The conducting shell used in the analysis is vertical on the inboard side, and approximately contouring the plasma boundary on the outboard side, with gaps at the top and bottom for the divertor. Fig. 14 shows this generic structure model, which is toroidally continuous. For the curves shown in Fig. 15, the conductor was tungsten, $0.035 \mathrm{~m}$ thick, with a resistivity of $8 \times 10^{-8} \mathrm{ohm}-\mathrm{m}$. In addition, the plasma has a $\beta_{p}$ of 0.25 and $l_{i}$ of 0.8 , typical of plasmas during rampup which are the most unstable. Fig. 15 shows that as the plasma elongation is increased the growth rate increases, and that the shell must be located closer to the plasma to provide any influence on the plasma. As the shell is moved further away, initially the growth rate changes slowly, but later begins increasing rapidly. Where this curve asymptotes is called the critical ideal wall location, and the stability factor is approaching 1.0. If the shell is located outside this location, it will not influence (slow down) the vertical instability even if it were superconducting. If the shell is located at this location or closer to the plasma, and it were superconducting it would stabilize the plasma. However, actual structural materials are resistive, so a more useful shell location is the critical resistive wall location. This is defined as the wall location that provides a stability factor of 1.2, and coincides with a shell location at the knee in the growth rate versus shell distance curve. Fig. 16 shows the stability factor for the same plasma elongations and wall distances with $f_{s}=1.2$ denoted on the plot.

The typical location for a conducting shell in power plant designs is between the blanket and the shield, which is roughly at a normalized distance of 0.45 times the minor radius, the precise value depending on the design. However, recent optimizations of the blanket for neutronics has allowed the conductor to be located inside the blanket, closer to the plasma. This location is found to be about 0.3-0.35 times the minor radius. For a shell at this location, elongations at the separatrix up to 2.2-2.3 can be considered, as opposed to those in ref[2] which could not exceed 1.9. The shells used in the scoping studies above are not practical since they surround the plasma and will adversely affect the neutronics. The shells are reduced in size by removing the section closest to the midplane, which has the weakest affect on vertical stability. This is done until the vertical stabilization provided by the shells starts to significantly degrade. The dark lines in Fig. 14 through the inboard and 
outboard shells show how much of the shells is removed for a final shell design, which in this case is $\pm 60^{\circ}$ on the outboard measured from the midplane. The inboard shell is reduced so that it has the same vertical extent as the outboard plate.

Once the plasma elongation is chosen, $\kappa$ (X-point $)=2.2$ in this case, and the final shell design is provided, more detailed vertical stability analysis is done to examine the impact of pressure and current profile on the growth rate. In addition, the vertical control calculations are done with this configuration to determine the maximum current and voltage expected during control, using the Tokamak Simulation Code[22]. It should be noted that since the stabilizing shells are located in the blanket they must operate at high temperatures and can not be actively cooled. The operating temperature turned out to be about $1100^{\circ} \mathrm{C}$. The resulting shell was 4.0 $\mathrm{cm}$ thick, and had a resistivity of about $35.0 \times 10^{-8} \mathrm{ohm}-\mathrm{m}$. Shown in Fig. 17 and 18 is the vertical instability growth rate as a function of $l_{i}$ and $\beta_{p}$, with the final reference plasma denoted by the $\times$. Also shown is the feedback control power (the product of peak current and peak voltage) for a random disturbance with $0.01 \mathrm{~m}$ RMS displacement of the plasma vertically, giving 30 MVA for the peak feedback power. Again, the reference plasma is denoted. The feedback power that can be tolerated determines the maximum vertical instability growth rate that is allowed, which is $45 / \mathrm{s}$. Consequently, the range of plasma pressure and current profiles that can be produced at full elongation is limited, and not to exceed the maximum growth rate. Plasmas with pressure and current profiles outside the range can be produced but only with lower elongation (which reduces the growth rate) or lower plasma current (which reduces the feedback power for a given growth rate). It should be noted that approximately $85 \%$ of the power for vertical position control is reactive, and therefore can be recovered with a suitable energy storage system, so that this power is not included in the recirculating power for the power plant.

\section{Poloidal Field Coil Optimization}

The poloidal field (PF) coil currents are determined to force the plasma boundary to pass through specified points in space, the desired outboard and inboard major radii, and produce a zero poloidal field at the desired $\mathrm{X}$-point. This is accomplished with a least-squares solution. Otherwise the plasma boundary is allowed to take on the shape that minimizes the coil stored energy. In addition, the PF coil locations are optimized to minimize an energy measure equal to the sum of the coil major radius times the coil current squared, which is found to scale with the coil cost. This is done by surrounding the plasma by a large number of PF coils, avoiding regions where coils can not exist (i.e. outboard midplane for maintenance). The coils are eliminated one by one and the resulting increase in the coil energy measure is determined. The coil that increases this measure the least is eliminated, and the process repeated with the remaining coils until a certain number of coils is obtained or the coil energy measure begins increasing rapidly. Fig. 19 shows the coil energy 
measure as function of the number of coils, and it begins to increase strongly below about 14 coils. It should be noted that there are 7 coils used to provide a thin solenoid on the inboard side, for inductive startup and plasma shaping, that are not included in the elimination. The final PF coil arrangement is shown in Fig. 20. The maintenance requires that entire sectors be removed through the outboard midplane, which forced the PF coils to be above $5.0 \mathrm{~m}$. The maximum current of 8.6 MA occurs in the largest radius coil, and Table 2 shows the coil locations and currents.

\section{Conclusions}

The advanced tokamak plasma configuration has the potential to provide a high $\beta$ and high bootstrap current fraction, resulting in a more compact economical power plant. The present work has utilized high resolution equilibrium and ideal MHD stability calculations in combination with a full velocity space bootstrap treatment to analize the impact of pressure profile and plasma shape optimization. In addition, plasma boundaries used in fixed boundary equilibria are provided by the $99 \%$ flux surface of the corresponding free-boundary equilibria.

Pressure profile optimization allowed the $\beta_{N}$ for ballooning instabilities to be maximized, while simultaneously providing bootstrap current alignment. Optimization of the plasma shape allowed the $\beta_{N}$ to be increased, but more importantly allowed the plasma current to be increased, leading to a $\beta$ nearly twice that in previous studies[2]. It was found that the minimum current drive requirement did not occur at the maximum $\beta$ due to a combination of bootstrap underdrive near the plasma edge and overdrive near the plasma center. The external kink mode is assumed to be stabilized by a combination of a conducting shell, located at 0.33 times the minor radius, and a feedback control system with coils located behind the shield. The vertical instability is slowed down by a conducting shell also located at 0.33 times the minor radius, and a feedback control coils located behind the shield. The resulting plasma configuration has a separatrix elongation and triangularity of 2.2 and 0.9 , respectively. The maximum $\beta$ is $10 \%$, with $q_{95} \geq 3.0$, resulting in a self-driven (bootstrap + diamagnetic + Phirsch-Schluter) current of $91 \%$. In order to provide margin between the operating pressure and the ideal MHD limit, the $\beta$ is reduced to $90 \%$ of its maximum value. The physics improvements noted here have made a significant contribution to the reduction in the power plant cost of electricity (COE) reported elsewhere[23], by increasing $\beta$ and reducing the external current drive power. 


\section{References}

[1] C. E. Kessel, J. Manickam, G. Rewoldt, and W. Tang, Improved Plasma Performance in Tokamaks with Negative Magnetic Shear, Phys. Rev. Lett., 72 (1994) 1212.

[2] S. C. Jardin, C. E. Kessel, C. G. Bathke, D. A. Ehst, T. K. Mau, F. Najmabadi, T. W. Petrie, and the ARIES Team, Physics Basis for a Reversed Shear Tokamak Power Plant, Fus. Engr. and Design, 38 (1997) 27.

[3] X. Litaudon, et al., Stationary Magnetic Shear Reversal Experiments in Tore Supra, Plas. Phys. Control. Fusion, 38 (1996) 1603.

[4] C. Gormezano, High Performance Tokamak Operation Regimes, Plas. Phys. Control. Fusion, 41 (1999) B367.

[5] A. Becoulet, et al., Performance and Control of Optimized Shear Discharges in JET, Nuc. Fus., 40 (2000) 1113.

[6] S. Ishida and the JT-60 Team, JT-60U High Performance Regimes, Nuc. Fus., 39 (1999) 1211.

[7] T. Oikawa and the JT-60 Team, High Performance and Prospects for Steady State Operation of JT-60U, Nuc. Fus., 40 (2000) 1125.

[8] V. S. Chan, et al., DIII-D Advanced Tokamak Research Overview, Nuc. Fus., $40(2000) 1137$.

[9] C. C. Petty, et al., Advanced Tokamak Physics in DIII-D, Plas. Phys. Control. Fusion, 42 (2000) B75.

[10] R. C. Wolf, et al., Stationary Advanced Scenarios with Internal Transport Barrier on ASDEX Upgrade, Plas. Phys. Control. Fusion, 41 (1999) B93.

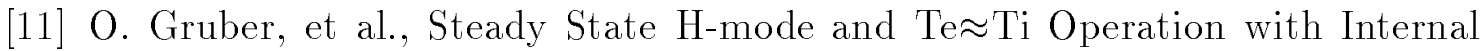
Transport Barriers in ASDEX Upgrade, Nuc. Fus., 40 (2000) 1145.

[12] J. Delucia, S. C. Jardin, and A. M. M. Todd, An Iterative Metric Method for Solving the Inverse Tokamak Equilibrium Problem, J. Comp. Phys., 37 (1980) 183.

[13] J. M. Greene and M. S. Chance, The Second Region of Stability Against Ballooning Modes, Nucl. Fusion, 21 (1981) 453.

[14] R. C. Grimm, R. L. Dewar, and J. Manickam, Ideal MHD Stability Calculations in Axisymmetric Toroidal Coordinate Systems, J. Comp. Phys., 49 (1983) 94. 
[15] S. W. Haney, L. D. Pearlstein, R. H. Bulmer, and J. P. Friedberg, Vertical Stability Analysis of Tokamaks Using a Variational Procedure, Plas. Phys. Reports, 23 (1997) 789.

[16] C. E. Kessel, Bootstrap Current in a Tokamak, Nuc. Fus., 34 (1994) 1221.

[17] S. C. Jardin, C. E. Kessel, T. K. Mau, et al., Physics Basis for the Advanced Tokamak Power Plant, Fus. Engr. and Design, this issue.

[18] S. P. Hirshman, Finite Aspect Ratio Effects on the Bootstrap Current in Tokamaks, Phys. Fluids, 31 (1988) 3150.

[19] L. Elguebaly, et al., Neutronics Analysis for ARIES-AT, Fus. Engr. and Design, this issue.

[20] A. Bondeson and D. J. Ward, Stabilization of External Modes in Tokamaks by Resistive Walls and Plasma Rotation, Phys. Rev. Lett., 72 (1994) 2709.

[21] Y. Q. Liu, A. Bondeson, C. M. Fransson, et al., Feedback Stabilization of NonAxisymmetric Resistive Wall Modes in Tokamaks. Part 1: Electromagnetic Model, Phys. Plas., 7 (2000) 3681.

[22] S. C. Jardin, N. Pomphrey, and J. Delucia, Dynamic Modeling of Transport and Position Control of Tokamaks, J. Comp. Phys., 66 (1986) 481.

[23] R. Miller, et al., Systems Analysis for ARIES-AT, Fus. Engr. and Design, this issue. 
Table 1:

ARIES-RS[2] and ARIES-AT Global Parameters

\begin{tabular}{|c|c|c|}
\hline & ARIES-RS & ARIES-AT \\
\hline$I_{p}(\mathrm{MA})$ & 11.3 & 12.8 \\
\hline$B_{T}(\mathrm{~T})$ & 7.98 & 5.86 \\
\hline $\mathrm{R}(\mathrm{m})$ & 5.52 & 5.20 \\
\hline $\mathrm{a}(\mathrm{m})$ & 1.38 & 1.30 \\
\hline$\kappa^{*}$ & 1.70 & 2.15 \\
\hline$\delta^{*}$ & 0.50 & 0.78 \\
\hline$\kappa(\mathrm{Xpt})$ & 1.90 & 2.20 \\
\hline$\delta(\mathrm{Xpt})$ & 0.70 & 0.90 \\
\hline$\beta_{p}$ & 2.29 & 2.28 \\
\hline$\beta(\%)$ & 4.98 & 9.07 \\
\hline$\beta_{*}(\%)$ & 6.18 & 11.0 \\
\hline$\beta_{N}(\%)$ & 4.84 & 5.40 \\
\hline$\beta_{N}^{\max }(\%)$ & 5.35 & 6.00 \\
\hline$q_{0}$ (axis) & 2.80 & 3.50 \\
\hline$q_{\min }(\operatorname{minimum})$ & 2.50 & 2.40 \\
\hline$q_{e}(\text { edge })^{*}$ & 3.52 & 3.70 \\
\hline$I_{b s}(\mathrm{MA})$ & 10.0 & 11.4 \\
\hline$I_{\nabla p} / I_{p}$ & 0.91 & 0.91 \\
\hline$I_{C D}(\mathrm{MA})$ & 1.15 & 1.25 \\
\hline$q_{*}$ & 2.37 & 1.85 \\
\hline$l_{i}(3)$ & 0.42 & 0.29 \\
\hline$n_{0} /\langle n\rangle$ & 1.36 & 1.34 \\
\hline$T_{o} /\langle T\rangle$ & 1.98 & 1.72 \\
\hline$p_{o} /\langle p\rangle$ & 2.20 & 1.93 \\
\hline$(b / a)_{k i n k}$ & 0.25 & 0.33 \\
\hline
\end{tabular}

*value corresponds to

fixed boundary equilibrium 


\section{ARIES-AT Poloidal Field Coil Parameters}

\begin{tabular}{lccc}
\hline coil \# & $\mathrm{R}(\mathrm{m})$ & $\mathrm{Z}(\mathrm{m})$ & $\mathrm{I}(\mathrm{MA})$ \\
\hline 1 & 2.25 & 0.25 & -0.620 \\
2 & 2.25 & 0.75 & -1.053 \\
3 & 2.25 & 1.25 & -1.513 \\
4 & 2.25 & 1.75 & -0.665 \\
5 & 2.25 & 2.25 & -0.665 \\
6 & 2.25 & 2.75 & 1.184 \\
7 & 2.25 & 3.25 & 3.360 \\
8 & 3.25 & 5.75 & 6.348 \\
9 & 3.75 & 6.00 & 6.518 \\
10 & 5.25 & 6.30 & 4.810 \\
11 & 5.75 & 6.25 & 3.643 \\
12 & 7.50 & 5.65 & -3.276 \\
13 & 8.00 & 5.40 & -5.877 \\
14 & 8.50 & 5.10 & -8.624 \\
\hline
\end{tabular}


Figure 1 - Equilibrium profiles for ARIES-AT, showing the plasma pressure, safety factor, parallel current, temperature, density, and poloidal flux.

Figure 2 - Variation of the plasma elongation and triangularity as one approaches the separatrix, where the elongation is 2.2 and triangularity is 0.9 .

Figure 3 - Comparison of collisionless (a) and collisional bootstrap (b) formulations for nearly $100 \%$ bootstrap fraction, showing that the collisionless form provides for a minimum safety factor that is closer to the plasma edge, resulting in no current drive requirement.

Figure 4 - A series of pressure gradient profiles as a function of poloidal flux showing how the gradient is reduced in the ballooning unstable region to obtain successively higher $\beta_{N}$ values, while keeping bootstrap alignment.

Figure 5 - Total externally driven current required as a function of $\beta_{N}$, showing that a minimum exists, and that the highest $\beta$ is not associated with the lowest current drive.

Figure $6-\beta_{N}$ as a function of the plasma triangularity.

Figure $7-\beta$ as a function of the plasma triangularity.

Figure $8-\beta_{N}$ as a function of the plasma elongation.

Figure $9-\beta$ as a function of the plasma elongation.

Figure 10 - Stabilizing wall location as a function of plasma elongation with the triangularity fixed at 0.7 , and for toroidal mode number from 1-6.

Figure 11 - Stabilizing wall location as a function of plasma elongation for the $\mathrm{n}=1$ kink mode, with various plasma triangularities.

Figure 12 - Stabilizing wall location as a function of toroidal mode number and various plasma triangularities, with fixed plasma elongation of 2.2.

Figure 13 - Stabilizing wall location as a function of toroidal mode number for two values of $\beta_{N}$, showing the shift of the limiting mode number as the pressure increases.

Figure 14 - Example of the generic structure used in the vertical stability calculations, and the final structure used in the design. 
Figure 15 - Vertical instability growth rate versus the distance of a tungsten shell, normalized to the minor radius, for various plasma elongations.

Figure 16 - Vertical stability factor as function of the distance of a tungsten shell, normalized to the minor radius, for various plasma elongations.

Figure 17 - Vertical instability growth rate as a function of the plasma internal self-inductance, $\operatorname{li}(3)$, and $\beta_{p}$, for the final design vertical stabilizing structure.

Figure 18 - Feedback power for vertical position control as a function of the vertical instability growth rate, for the final vertical stabilizing structure.

Figure 19 - Poloidal field coil energy as function of the number of coils, showing that the energy increases as the number of coils decreases.

Figure 20 - Layout of the optimized poloidal field coils. 

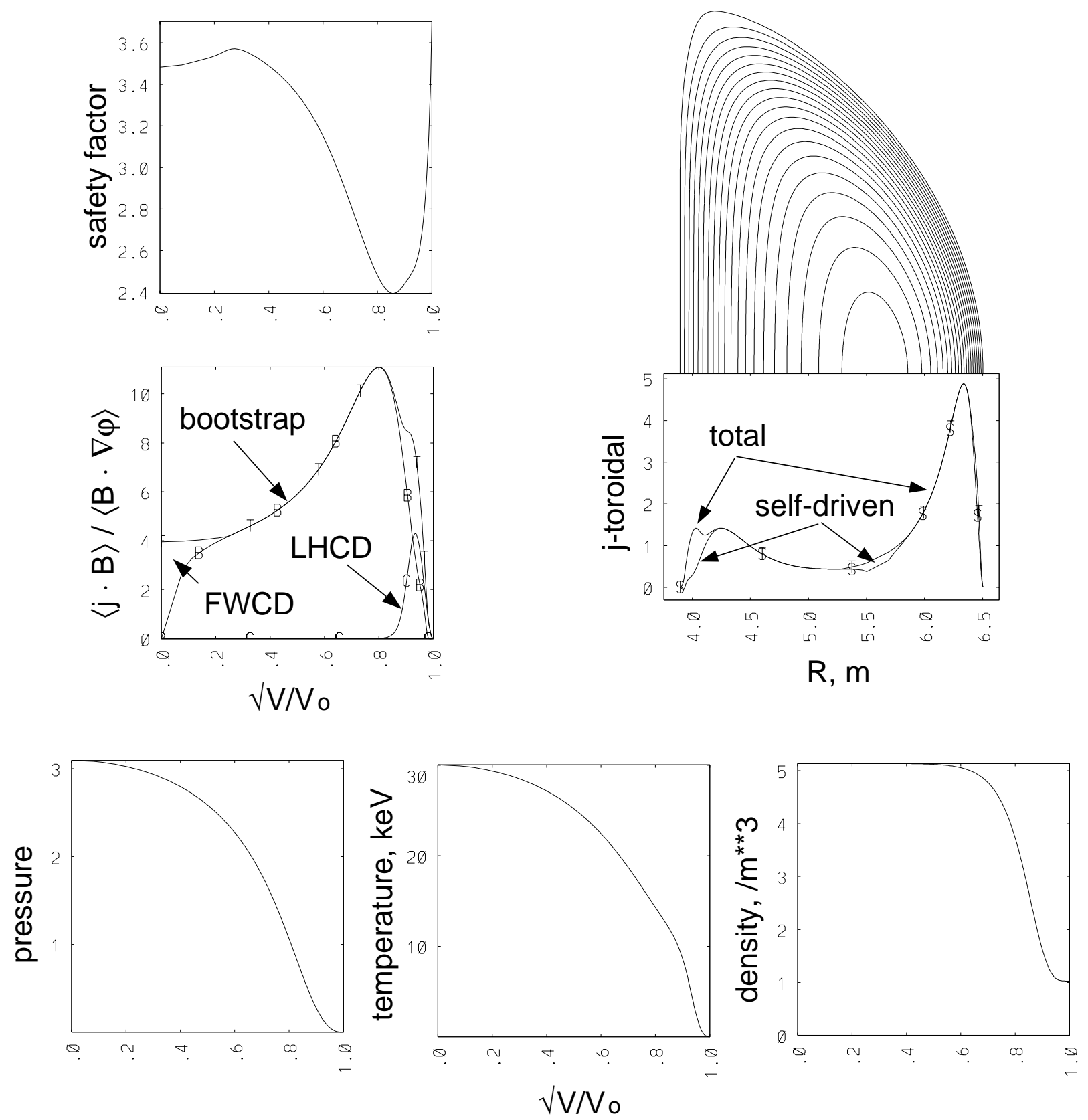

Figure 1: 


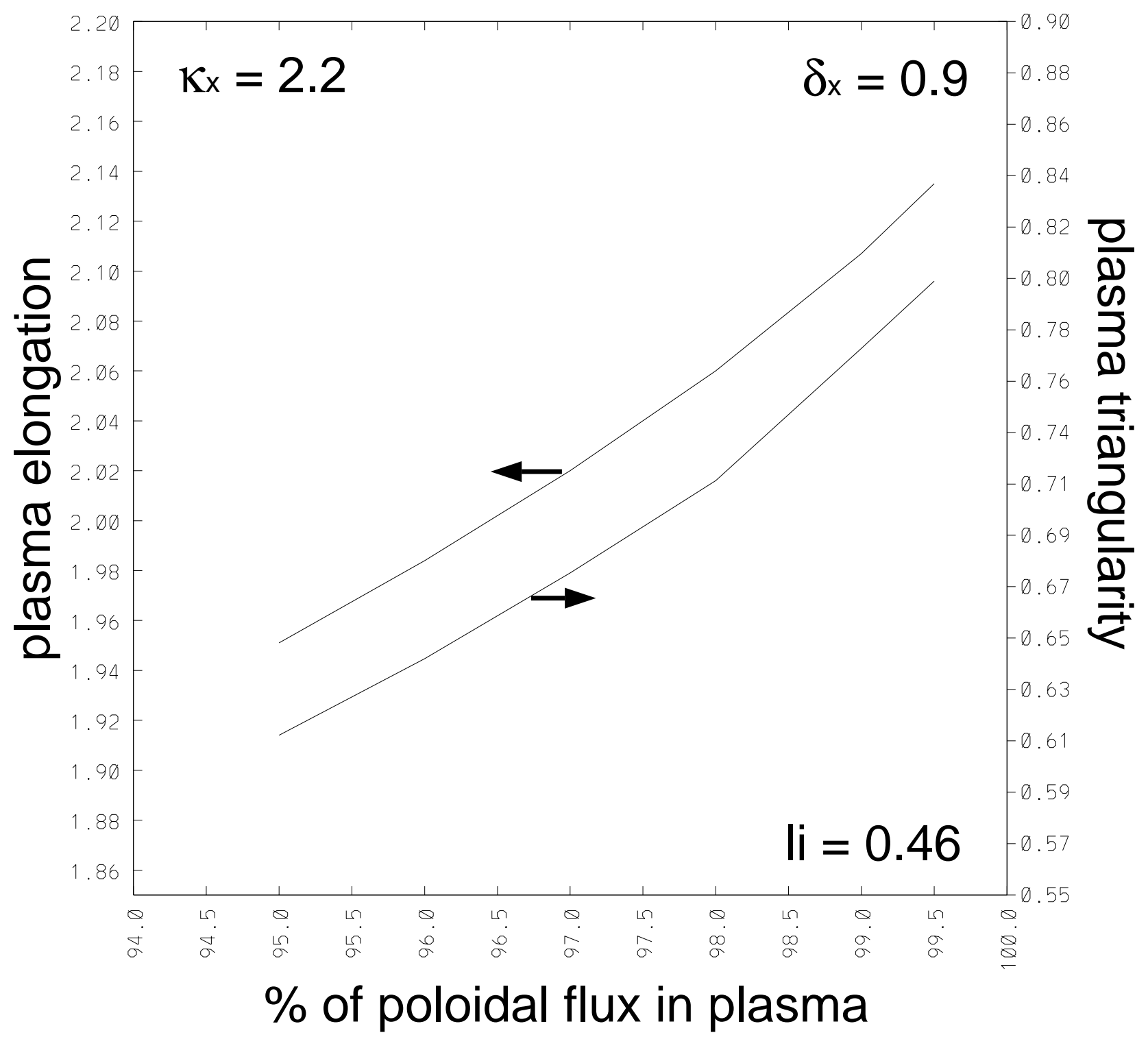

Figure 2: 

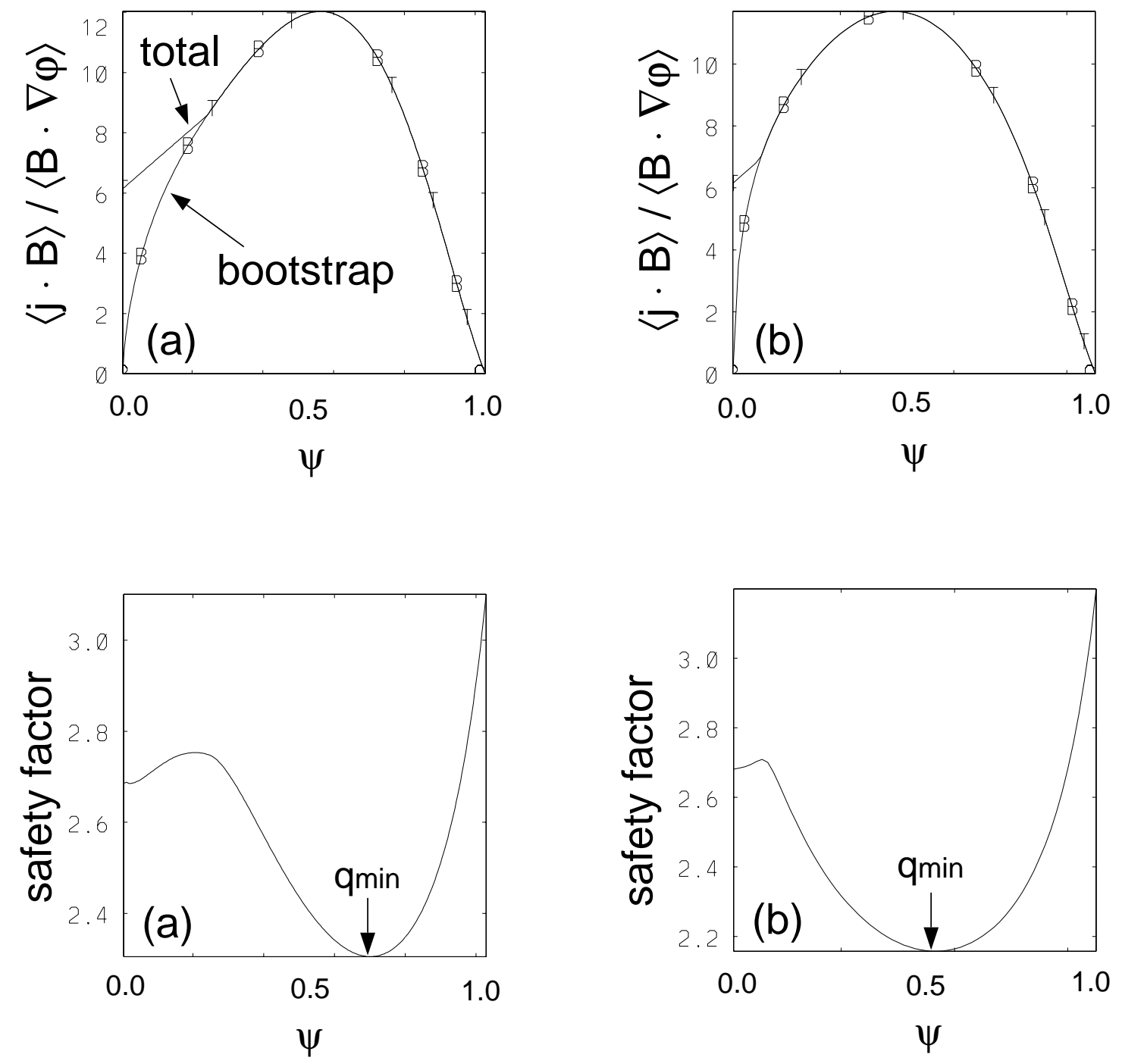

Figure 3: 


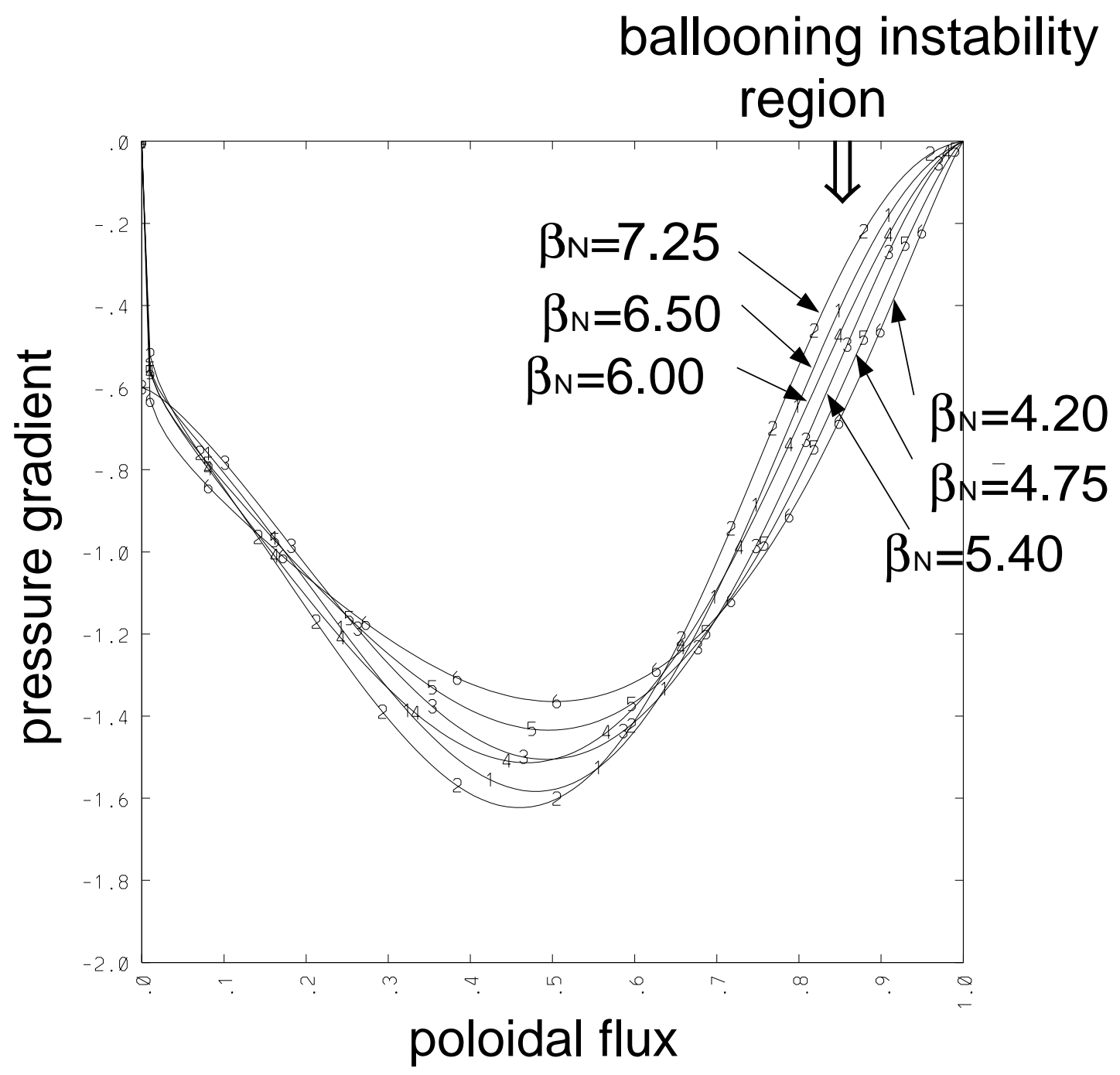

Figure 4: 


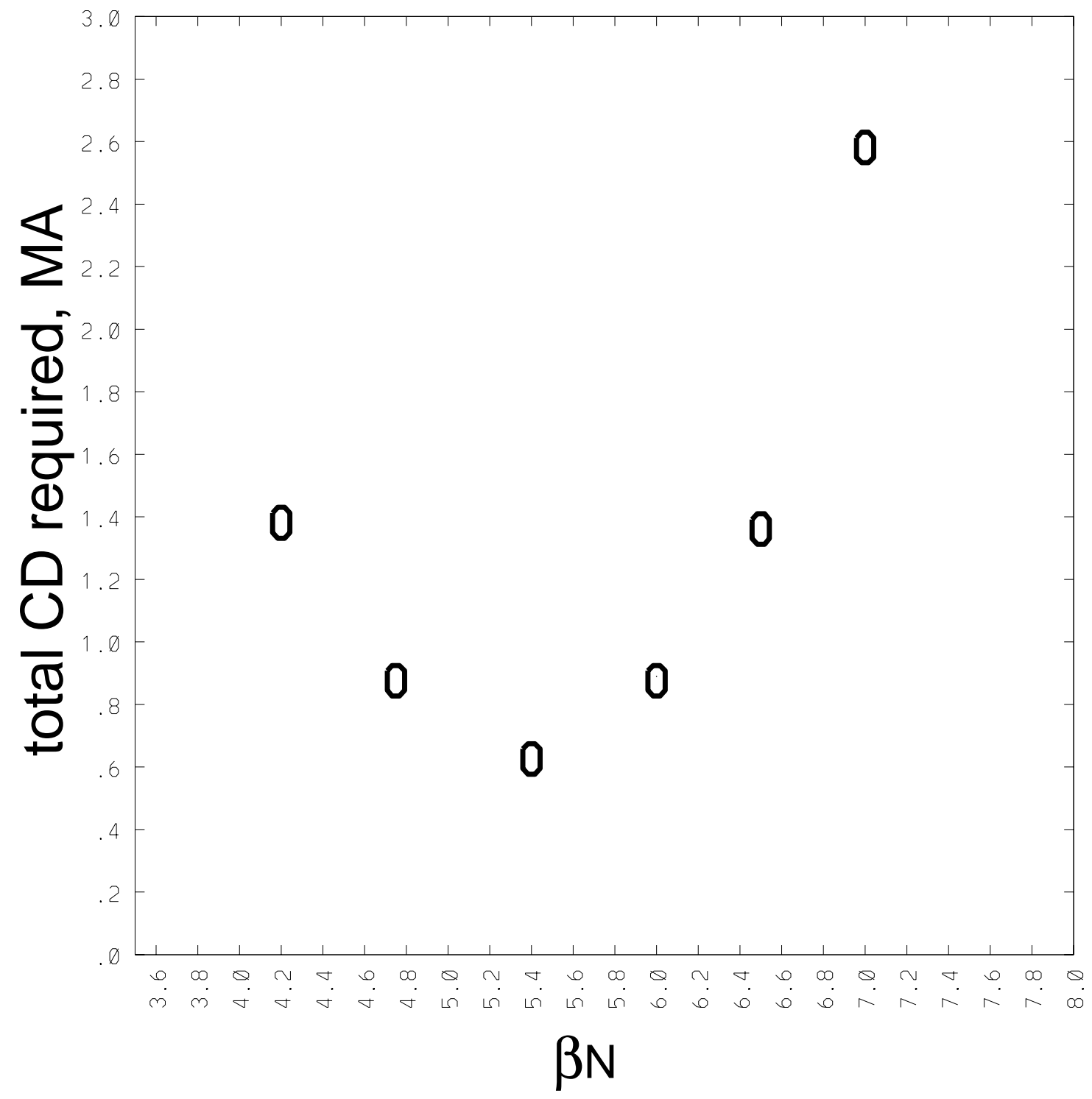

Figure 5: 


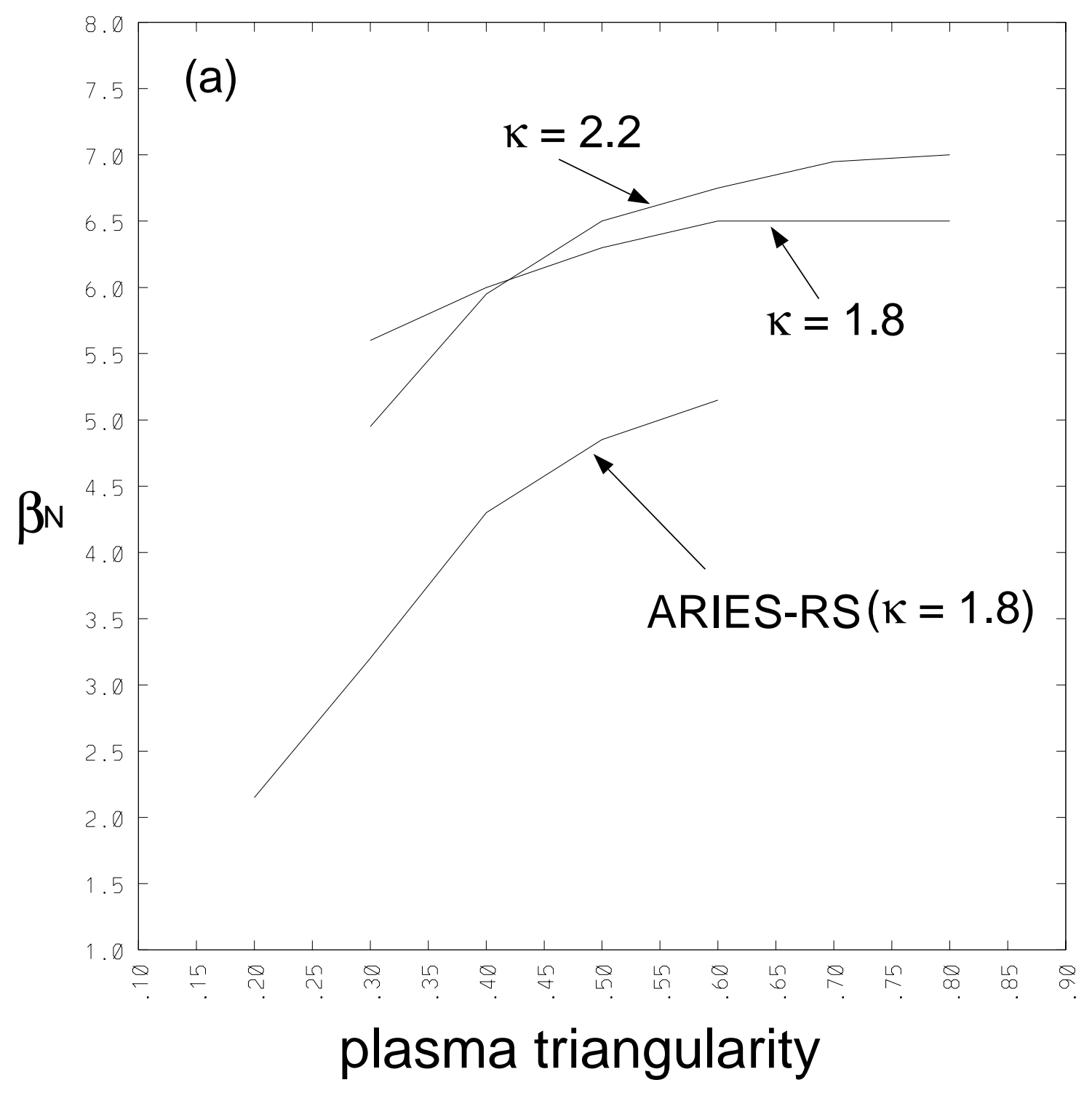

Figure 6: 


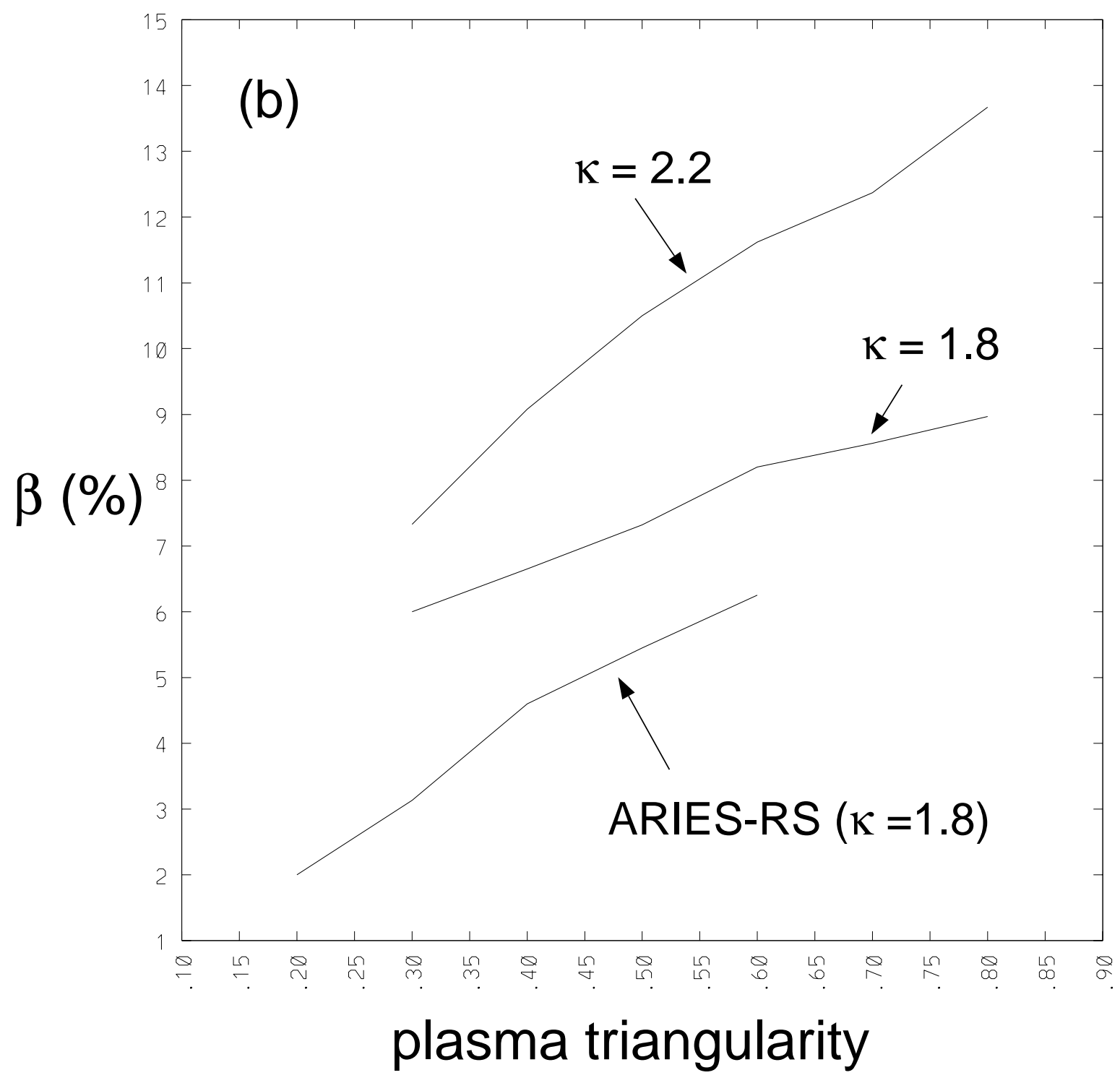

Figure 7: 


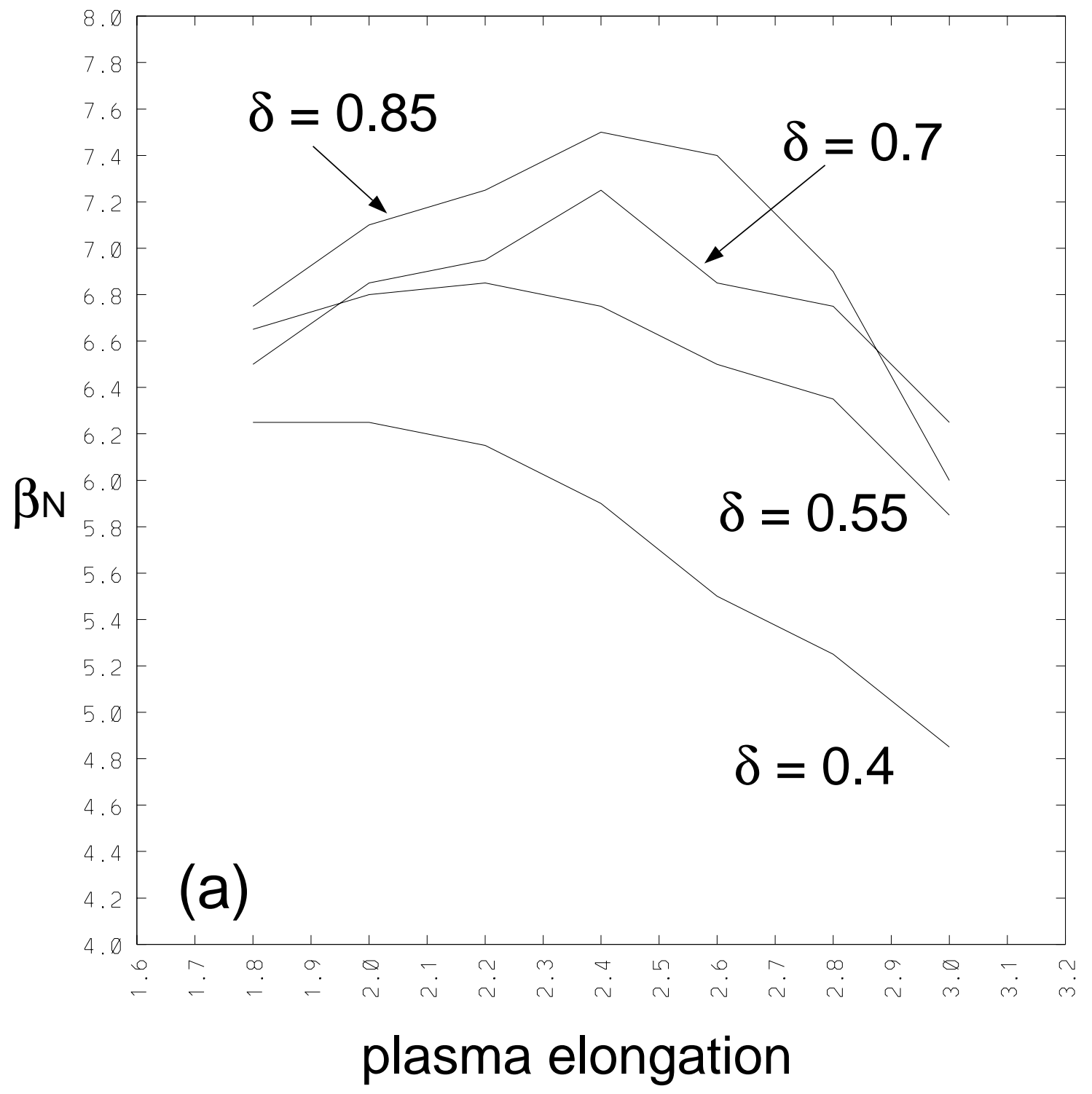

Figure 8: 


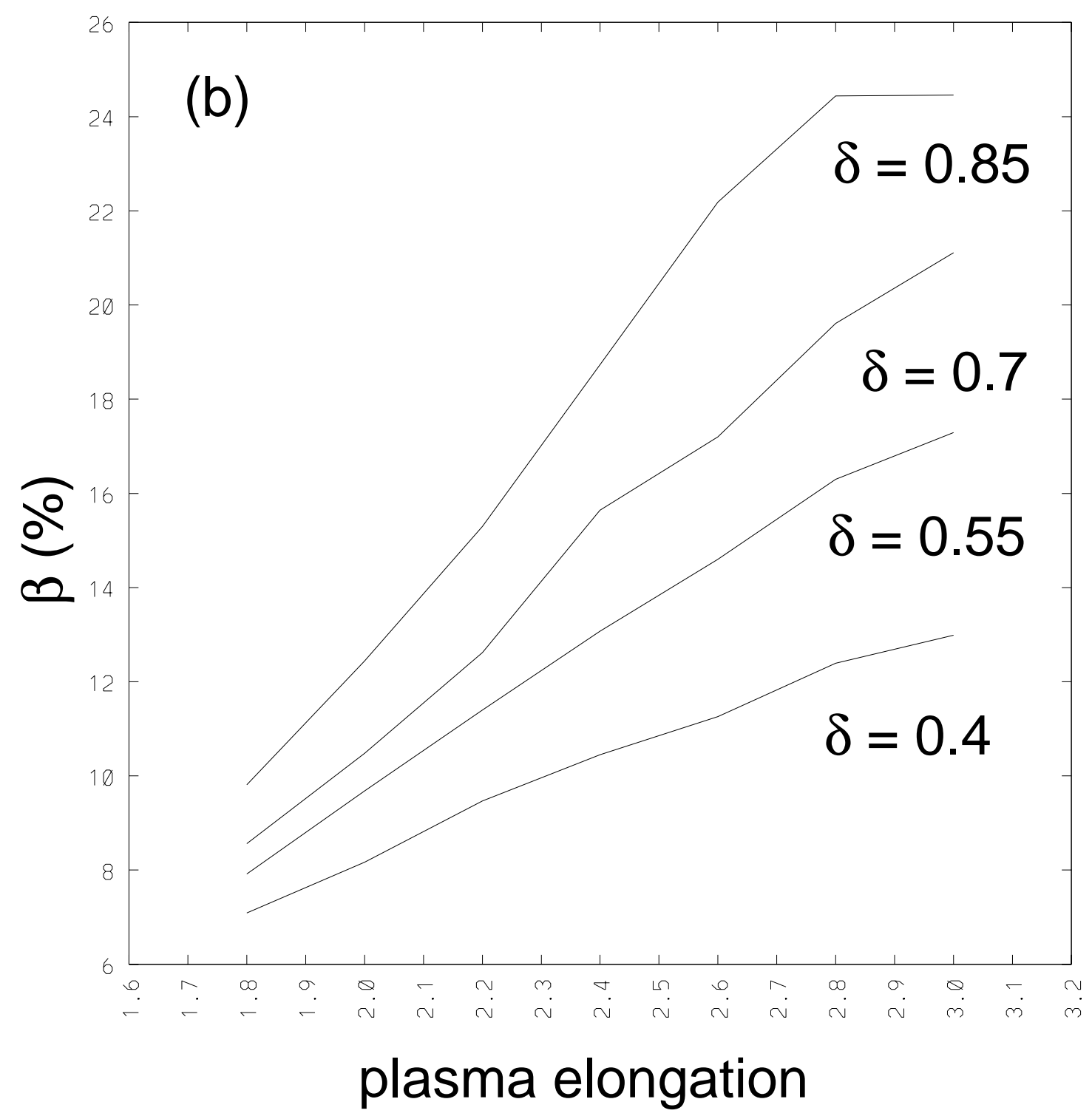

Figure 9: 


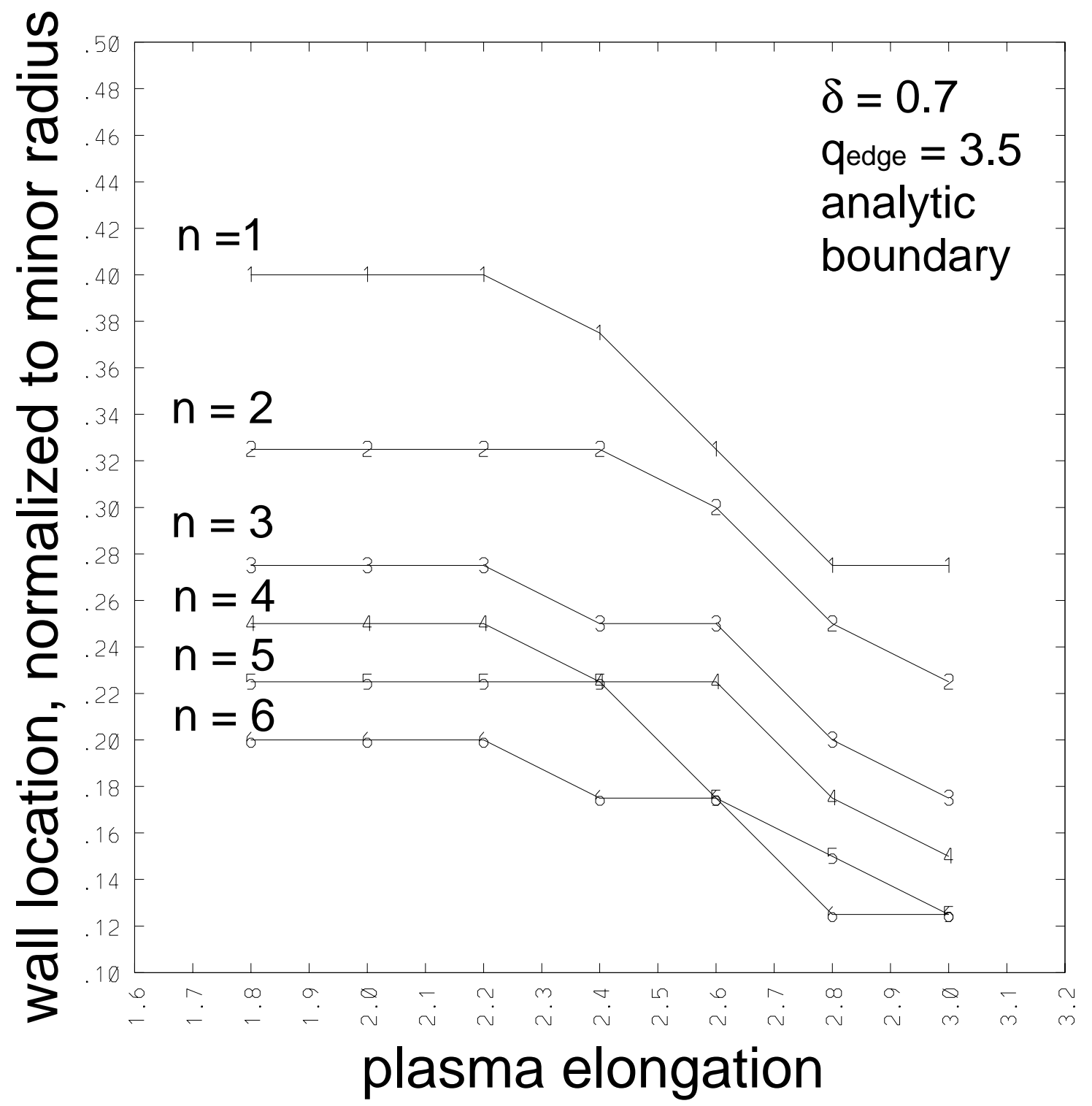

Figure 10: 


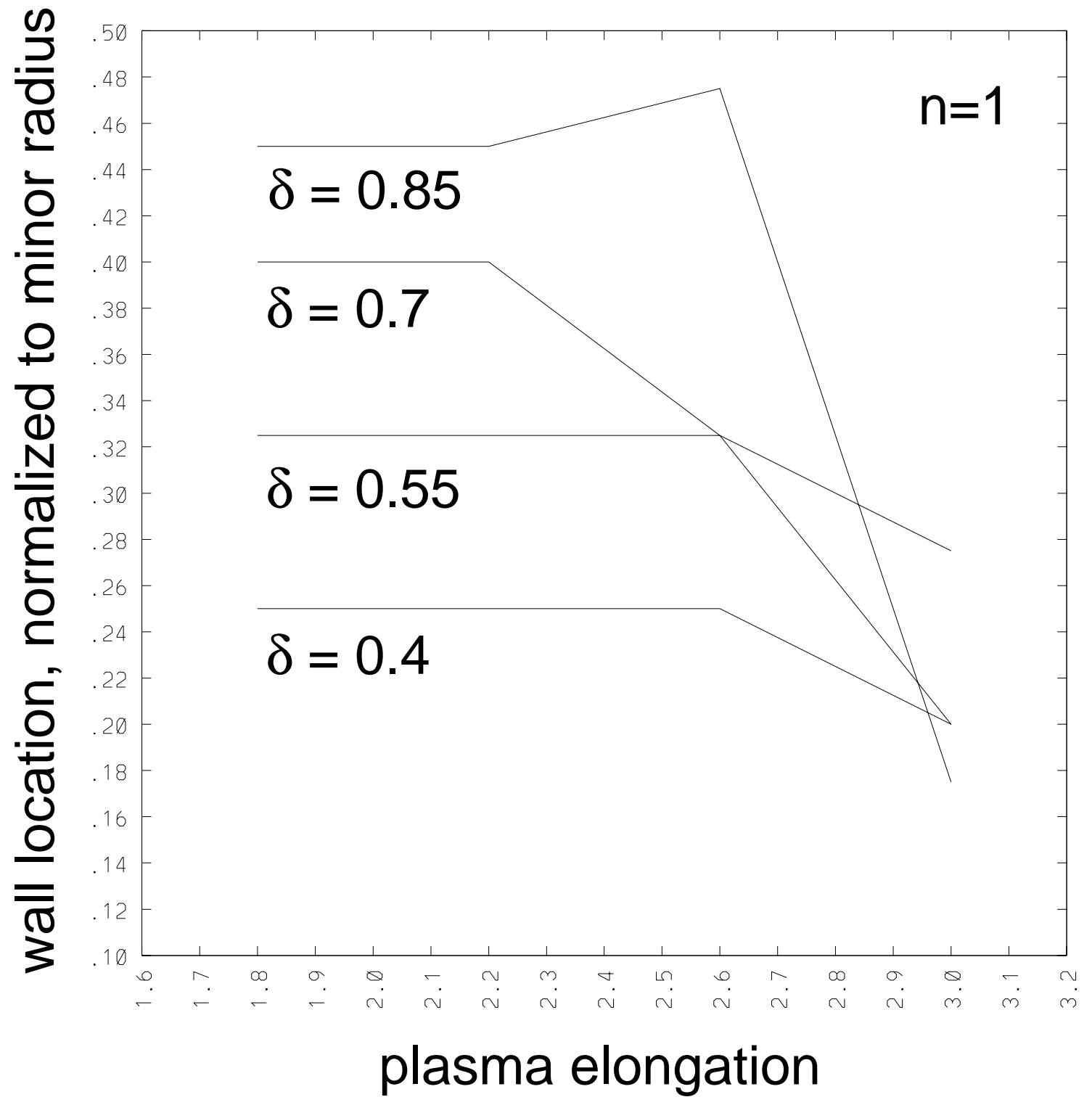

Figure 11: 


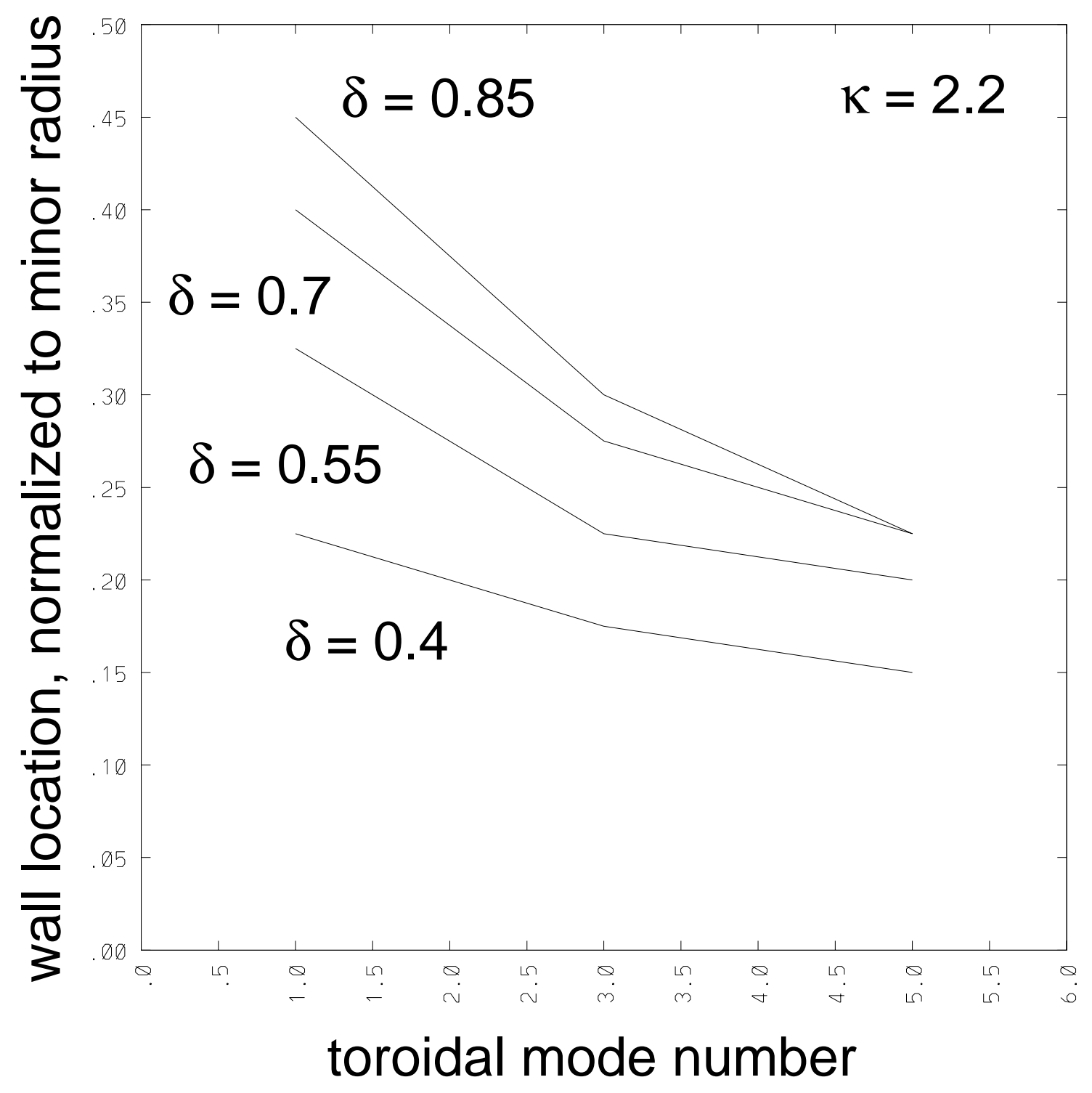

Figure 12: 


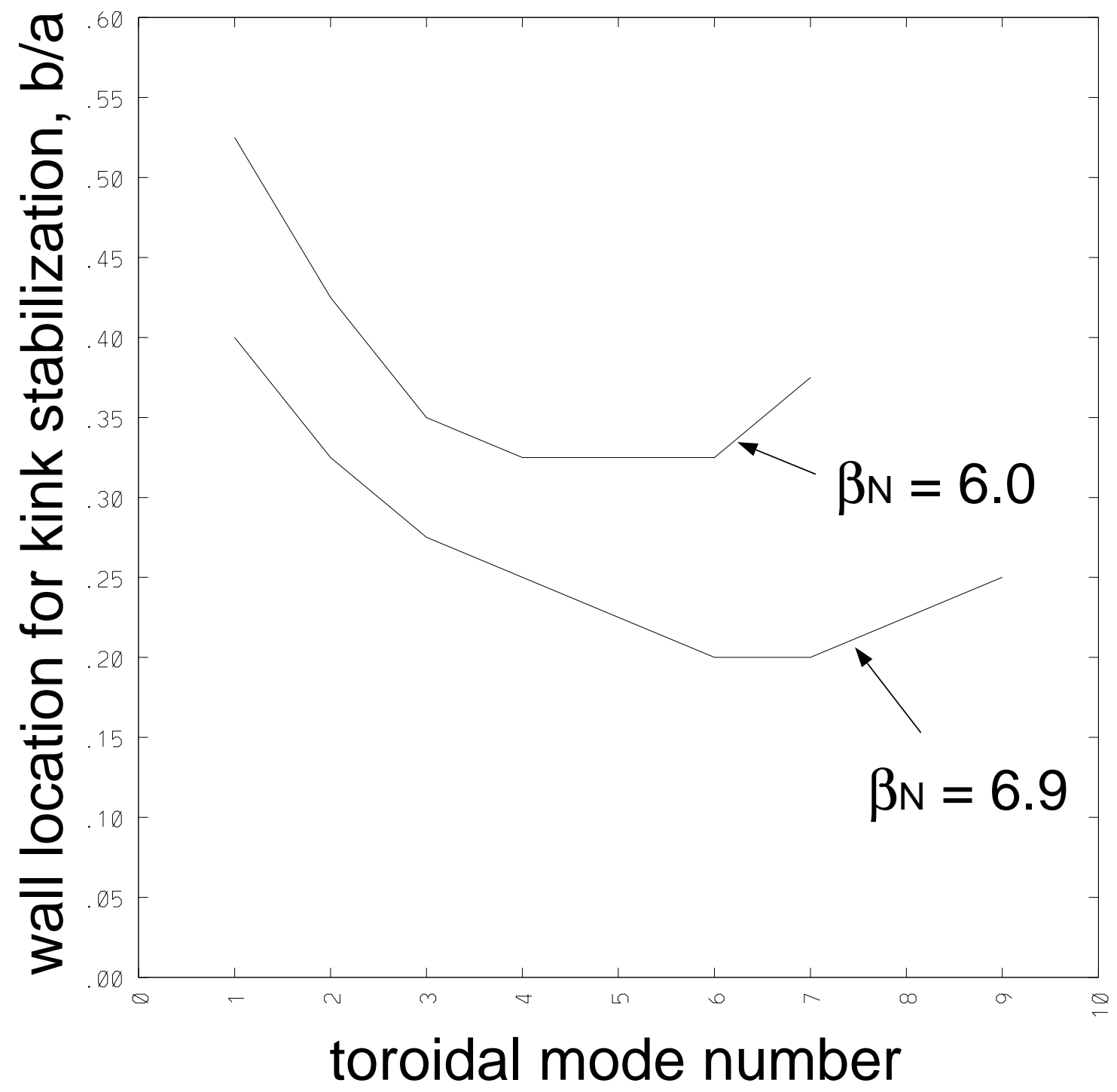

Figure 13: 


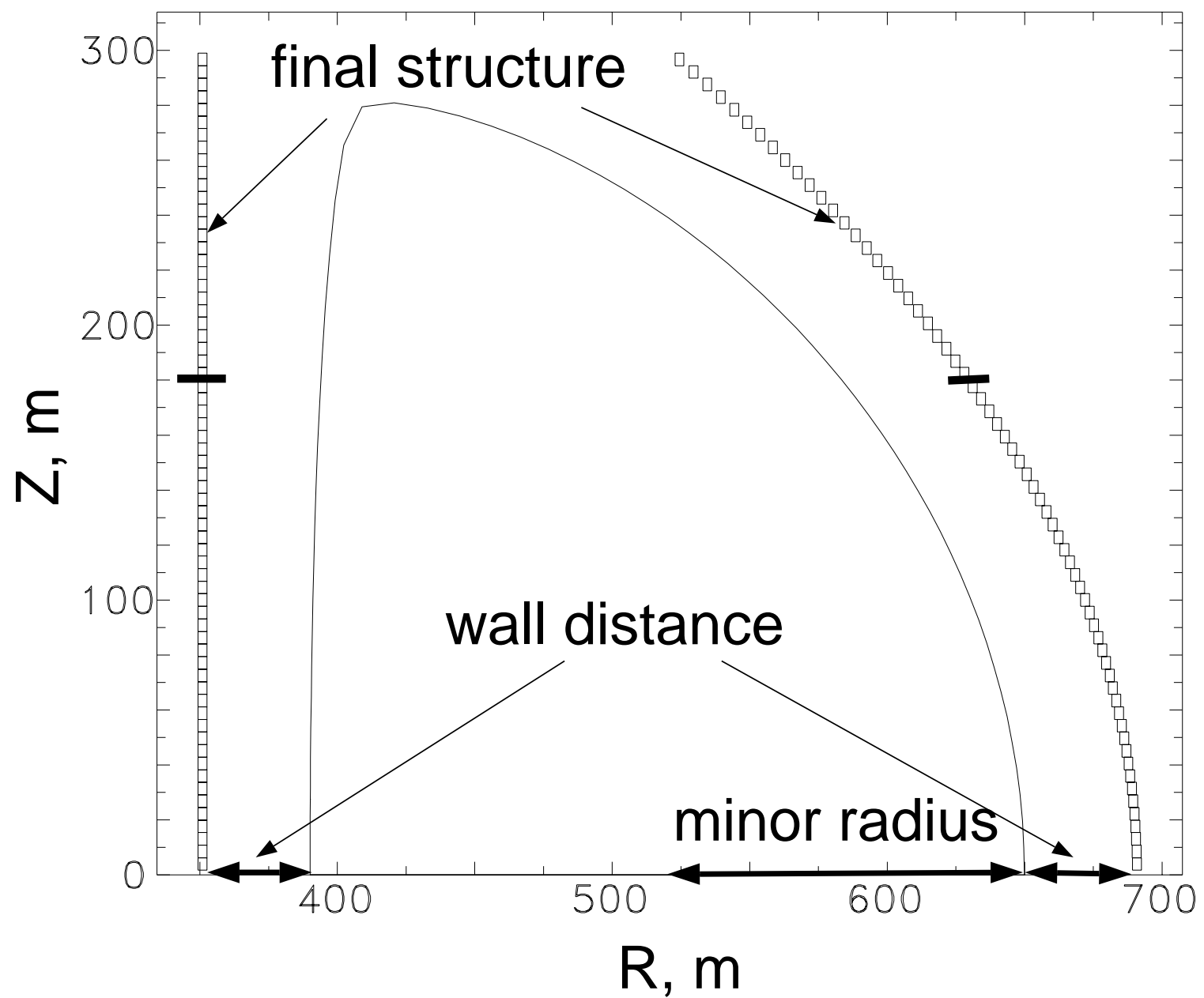

Figure 14: 


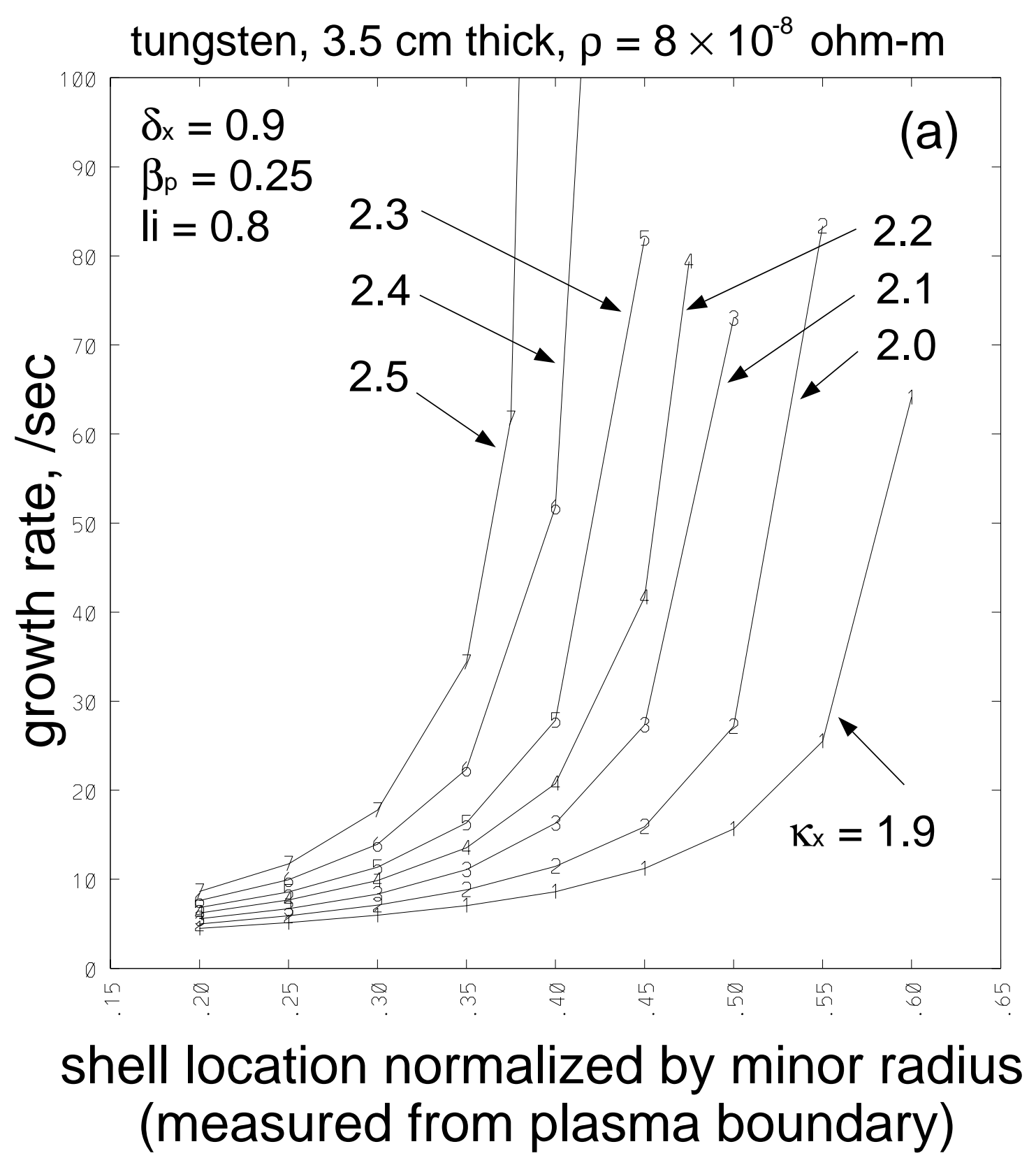

Figure 15: 


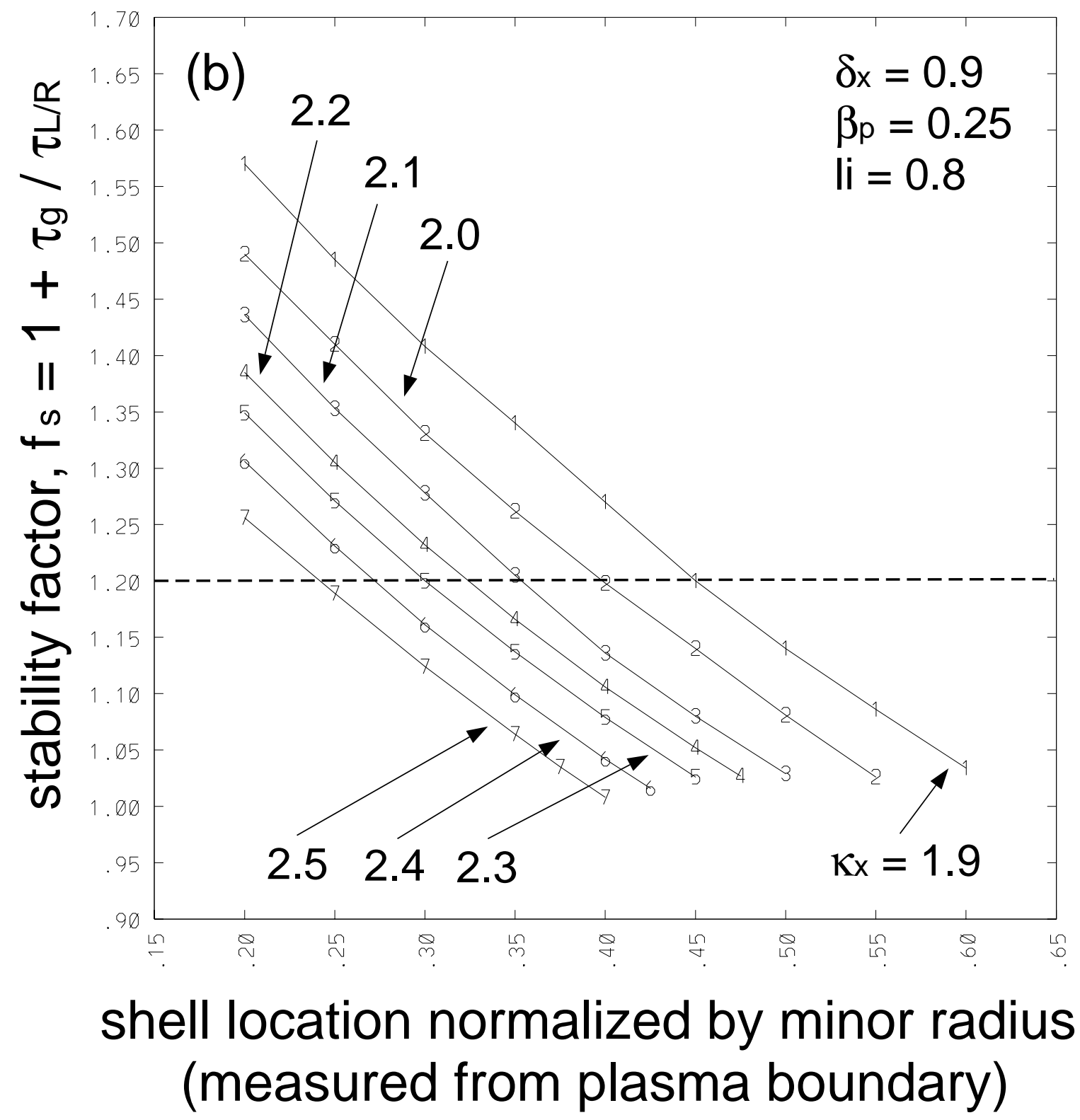

Figure 16: 


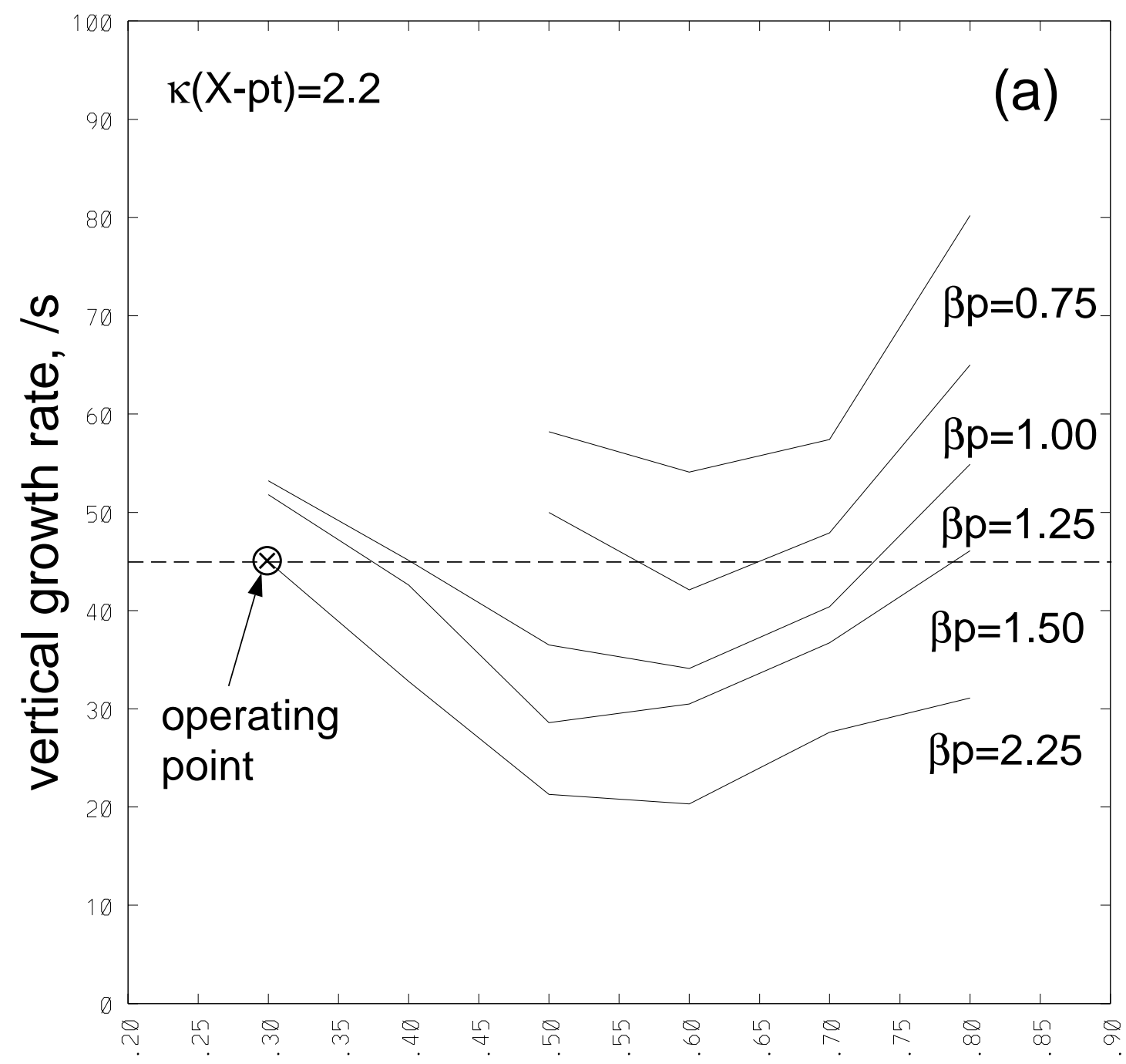

plasma internal inductance, li(3)

Figure 17: 


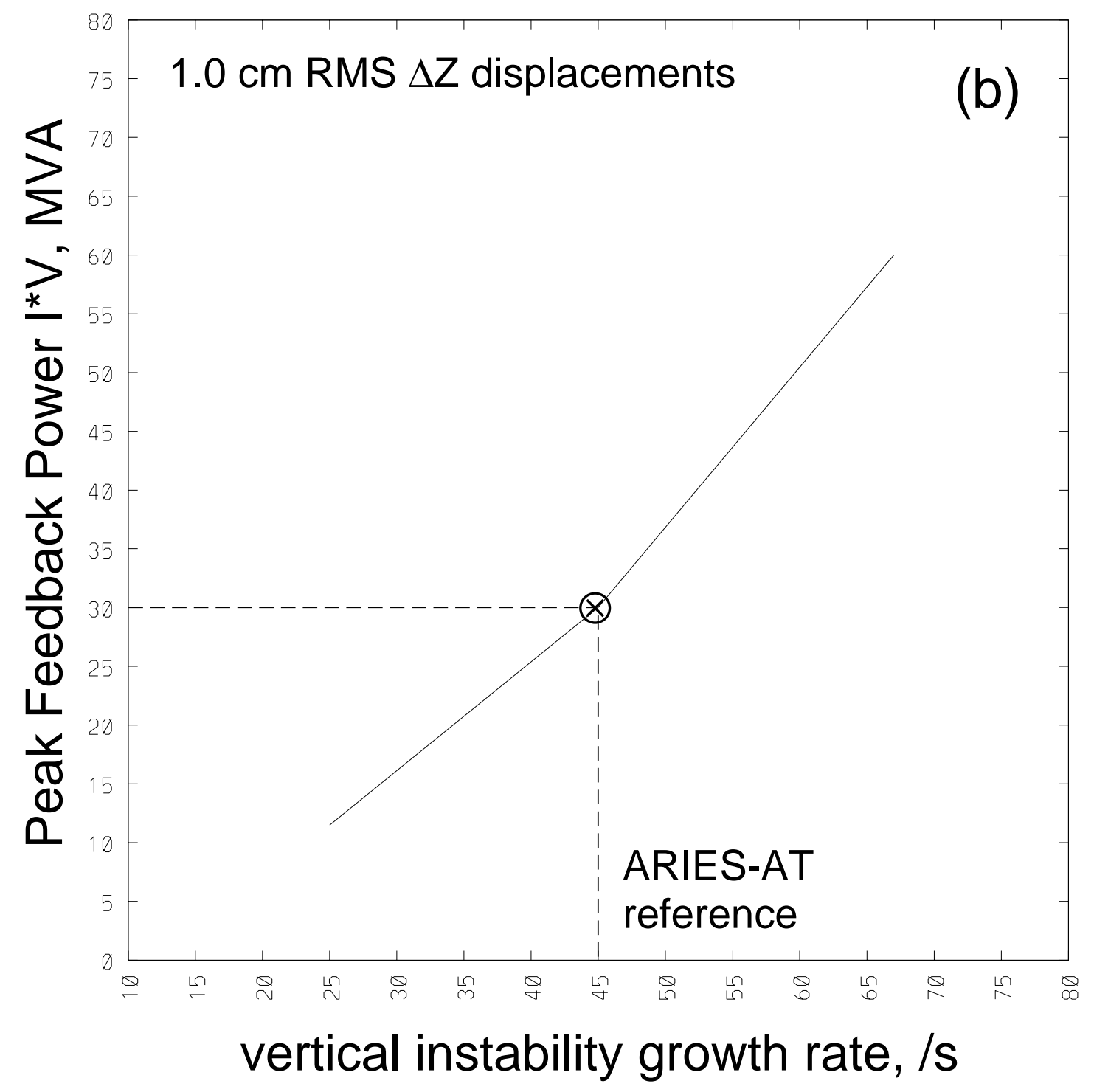

Figure 18: 


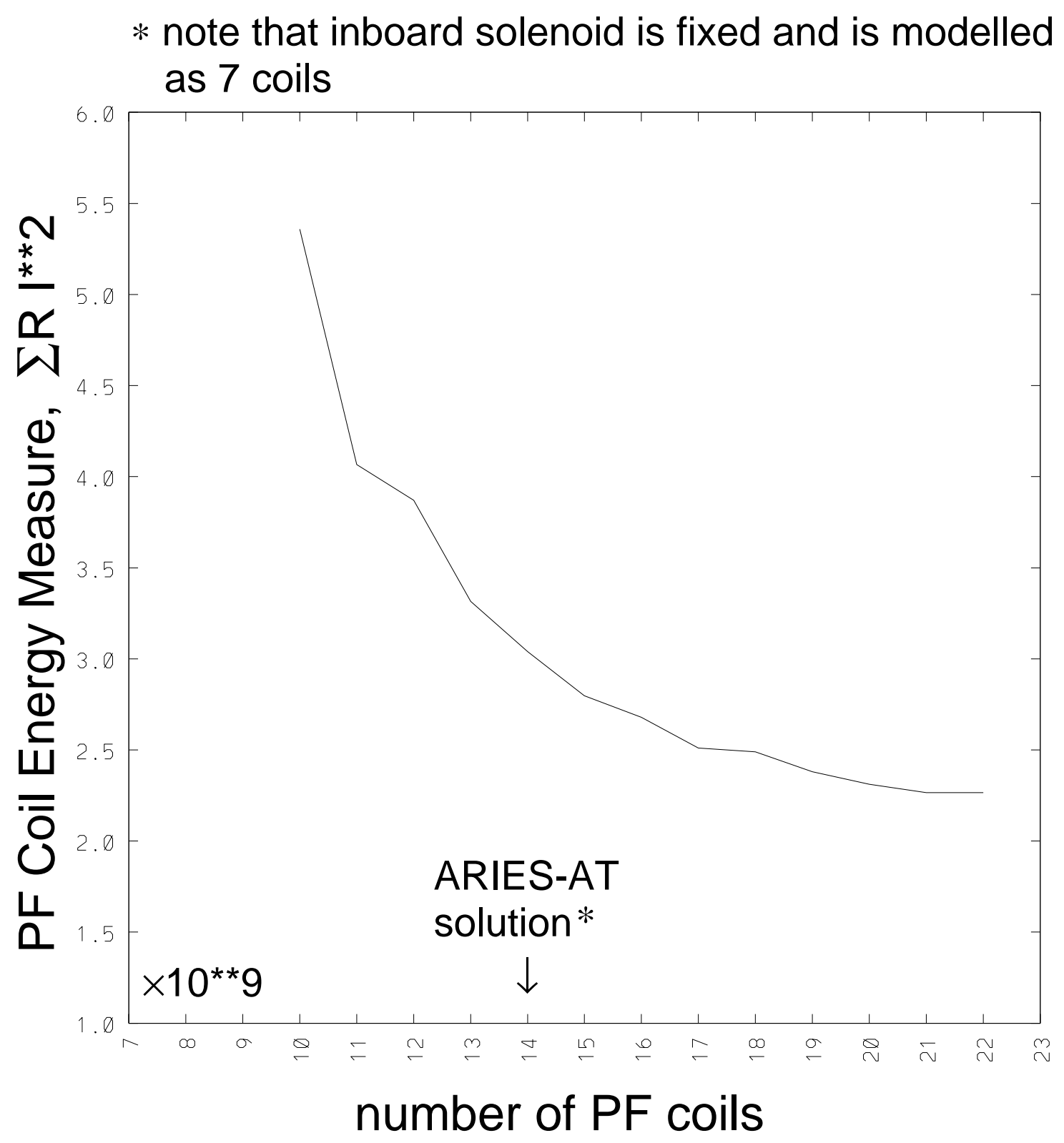

Figure 19: 


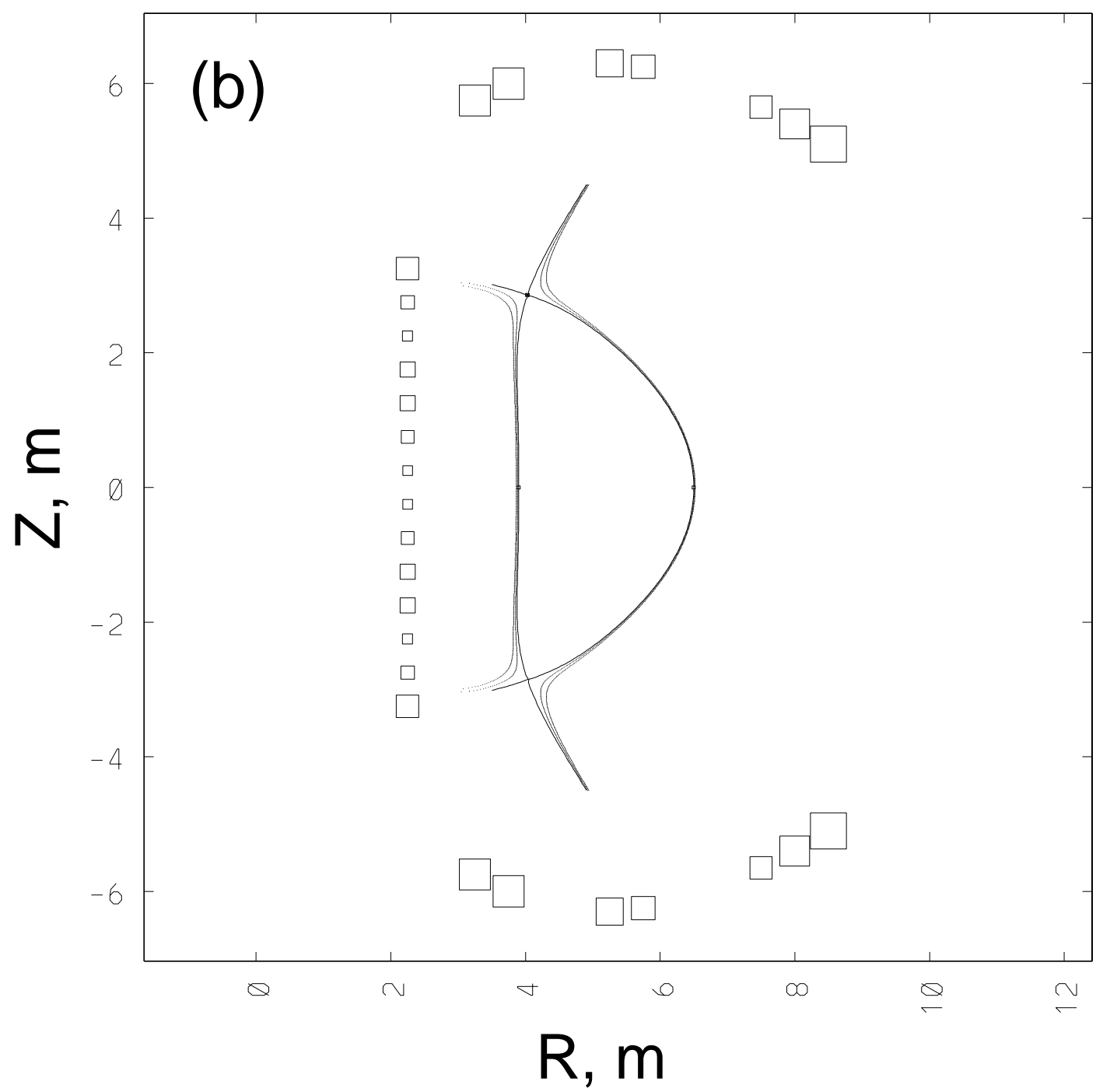

Figure 20: 


\section{External Distribution}

Plasma Research Laboratory, Australian National University, Australia

Professor I.R. J ones, Flinders University, Australia

Professor J oão Canalle, Instituto de Fisica DEQ/IF - UERJ , Brazil

Mr. Gerson O. Ludwig, Instituto Nacional de Pesquisas, Brazil

Dr. P.H. Sakanaka, Instituto Fisica, Brazil

The Librarian, Culham Laboratory, England

Library, R61, Rutherford Appleton Laboratory, England

Mrs. S.A. Hutchinson, JET Library, England

Professor M.N. Bussac, Ecole Polytechnique, France

Librarian, Max-Planck-Institut für Plasmaphysik, Germany

J olan Moldvai, Reports Library, MTA KFKI-ATKI, Hungary

Dr. P. Kaw, Institute for Plasma Research, India

Ms. P.J . Pathak, Librarian, Insitute for Plasma Research, India

Ms. Clelia De Palo, Associazione EURATOM-ENEA, I taly

Dr. G. Grosso, Instituto di Fisica del Plasma, Italy

Librarian, Naka Fusion Research Establishment, J AERI, J apan

Library, Plasma Physics Laboratory, Kyoto University, J apan

Research Information Center, National Institute for Fusion Science, J apan

Dr. O. Mitarai, Kyushu Tokai University, J apan

Library, Academia Sinica, Institute of Plasma Physics, People's Republic of China

Shih-Tung Tsai, Institute of Physics, Chinese Academy of Sciences, People's Republic of China

Dr. S. Mirnov, TRINITI, Troitsk, Russian Federation, Russia

Dr. V.S. Strelkov, Kurchatov Institute, Russian Federation, Russia

Professor Peter Lukac, Katedra Fyziky Plazmy MFF UK, Mlynska dolina F-2, Komenskeho Univerzita, SK-842 15 Bratislava, Slovakia

Dr. G.S. Lee, Korea Basic Science Institute, South Korea

Mr. Dennis Bruggink, Fusion Library, University of Wisconsin, USA

Institute for Plasma Research, University of Maryland, USA

Librarian, Fusion Energy Division, Oak Ridge National Laboratory, USA

Librarian, Institute of Fusion Studies, University of Texas, USA

Librarian, Magnetic Fusion Program, Lawrence Livermore National Laboratory, USA

Library, General Atomics, USA

Plasma Physics Group, Fusion Energy Research Program, University of California at San Diego, USA

Plasma Physics Library, Columbia University, USA

Alkesh Punjabi, Center for Fusion Research and Training, Hampton University, USA

Dr. W.M. Stacey, Fusion Research Center, Georgia Institute of Technology, USA

Dr. J ohn Willis, U.S. Department of Energy, Office of Fusion Energy Sciences, USA

Mr. Paul H. Wright, Indianapolis, Indiana, USA 
The Princeton Plasma Physics Laboratory is operated by Princeton University under contract with the U.S. Department of Energy.

\author{
Information Services \\ Princeton Plasma Physics Laboratory \\ P.O. Box 451 \\ Princeton, NJ 08543
}

Phone: 609-243-2750

Fax: 609-243-2751

e-mail: pppl_info@pppl.gov

Internet Address: http://www.pppl.gov 